Article

\title{
Sensitivity of High-Scale SUSY in Low Energy Hadronic FCNC
}

\author{
Morimitsu Tanimoto $^{1, *}$ and Kei Yamamoto $^{2}$ \\ ${ }^{1}$ Department of Physics, Niigata University, Niigata 950-2181, Japan \\ ${ }^{2}$ High Energy Accelerator Research Organization (KEK) Theory Center, Institute of Particle and \\ Nuclear Studies (IPNS), KEK, Tsukuba, Ibaraki 305-0801, Japan; \\ E-Mail: yamamoto@muse.sc.niigata-u.ac.jp \\ * Author to whom correspondence should be addressed; E-Mail: tanimoto@ muse.sc.niigata-u.ac.jp; \\ Tel.: +81-25-262-6191.
}

Academic Editors: Shin'ichi Nojiri, Sergei Odintsov and Jonathan Bougie

Received: 6 April 2015 / Accepted: 14 May 2015 / Published: 21 May 2015

\begin{abstract}
We discuss the sensitivity of the high-scale supersymmetry (SUSY) at 10-1000 TeV in $B^{0}, B_{s}, K^{0}$ and $D$ meson systems together with the neutron electric dipole moment (EDM) and the mercury EDM. In order to estimate the contribution of the squark flavor mixing to these flavor changing neutral currents (FCNCs), we calculate the squark mass spectrum, which is consistent with the recent Higgs discovery. The SUSY contribution in $\epsilon_{K}$ could be large, around $40 \%$ in the region of the SUSY scale 10-100 TeV. The neutron EDM and the mercury EDM are also sensitive to the SUSY contribution induced by the gluino-squark interaction. The predicted EDMs are roughly proportional to $\left|\epsilon_{K}^{\mathrm{SUSY}}\right|$. If the SUSY contribution is the level of $\mathcal{O}(10 \%)$ for $\epsilon_{K}$, the neutron EDM is expected to be discovered in the region of $10^{-28}-10^{-26} \mathrm{ecm}$. The mercury EDM also gives a strong constraint for the gluino-squark interaction. The SUSY contribution of $\Delta M_{D}$ is also discussed.
\end{abstract}

Keywords: supersymmetry; high-scale supersymmetry (SUSY); flavor changing neutral current (FCNC); neutron electric dipole moment (EDM)

\section{Introduction}

Supersymmetry (SUSY) is one of the most attractive theories beyond the standard model (SM). Therefore, SUSY has been expected to be observed at the LHC experiments. However, no signals of 
SUSY have been discovered yet. The present searches for SUSY particles give us important constraints for SUSY. Since the lower bounds of the superparticle masses increase gradually, the squark and the gluino masses are supposed to be at a higher scale than $1 \mathrm{TeV}$ [1-3]. On the other hand, the SUSY model has been seriously constrained by the Higgs discovery, in which the Higgs mass is $125 \mathrm{GeV}$ [4-6]. Based on this theoretical and experimental situation, we consider the high-scale SUSY models, which have been widely discussed with a great deal of attention [7-22].

If the squark and slepton masses are at the high-scale $\mathcal{O}(10-1000) \mathrm{TeV}$, the lightest Higgs mass can be pushed up to $125 \mathrm{GeV}$, whereas SUSY particles are out of the reach of the LHC experiment. Therefore, the indirect search of the SUSY particles becomes important in the low-energy flavor physics [23-25].

The flavor physics is also on the a stage in light of $\mathrm{LHCb}$ data. The LHCb collaboration has reported new data of the $\mathrm{CP}$ violation of the $B_{s}$ meson and the branching ratios of rare $B_{s}$ decays [26-38]. For many years, the $\mathrm{CP}$ violation in the $K$ and $B^{0}$ mesons has been successfully understood within the framework of the standard model (SM), the so-called Kobayashi-Maskawa (KM) model [39], where the source of the $\mathrm{CP}$ violation is the $\mathrm{KM}$ phase in the quark sector with three families. However, a new physics has been expected to be indirectly discovered in the precise data of $B^{0}$ and $B_{s}$ meson decays at the $\mathrm{LHCb}$ experiment and the further coming experiment, Belle-II.

There are new sources of the CP violation if the SM is extended to the SUSY models. The soft squark mass matrices contain the $\mathrm{CP}$ violating phases, which contribute to the flavor changing neutral current (FCNC) with the CP violation [40]. Therefore, we can expect the SUSY effect in the CP violating phenomena. However, the clear deviation from the SM prediction has not been observed yet in the LHCb experiment [26-38]. Actually, we have found that the CP violation of $B^{0}$ and $B_{s}$ meson systems are suppressed if the SUSY scale is above $10 \mathrm{TeV}$ [41]. On the other hand, the CKM fitter group presented the current limits on new physics contributions of $\mathcal{O}(10 \%)$ in $B^{0}, B_{s}$ and $K^{0}$ systems [42]. They have also estimated the sensitivity to new physics in $B^{0}$ and $B_{s}$ mixing achievable with $50 \mathrm{ab}^{-1}$ of Belle-II and $50 \mathrm{fb}^{-1}$ of LHCb data. Therefore, we should carefully study the sensitivity of the high-scale SUSY to the hadronic FCNC.

In this work, we discuss the high-scale SUSY contribution to the $B^{0}, B_{s}$ and $K^{0}$ meson systems. Furthermore, we also discuss the sensitivity to the $D$ meson and the electric dipole moment (EDM) of the neutron and mercury. For these modes, the most important process of the SUSY contribution is the gluino-squark-mediated flavor changing process [43-58]. The CP violation of the $K$ meson, $\epsilon_{K}$, provides a severe constraint to the gluino-squark-mediated FCNC [59,60]. In addition, recent work has found that the chromo-electric dipole moment (cEDM) is sensitive to the high-scale SUSY [61]. It is noted that the upper-bound of the neutron EDM (nEDM) [62] gives a severe constraint for the gluino-squark interaction through the cEDM [63-68]. It is also remarked that the upper bound of the mercury EDM (HgEDM) [69] can give an important constraint [70].

In order to estimate the gluino-squark-mediated FCNC of the $K, B^{0}, B_{s}$ and $D$ mesons, we work in the basis of the squark mass eigenstate with the non-minimal squark (slepton) flavor mixing. There are three reasons why the SUSY contribution to the FCNC considerably depends on the squark mass spectrum. The first one is that the GIM mechanism works in the squark flavor mixing, and the second one is that the loop functions depend on the mass ratio of the squark and gluino. The last one is that we need the mixing angle between the left-handed sbottom and right-handed sbottom, which dominates the 
$\Delta B=1$ decay processes. Therefore, we discuss the squark mass spectrum, which is consistent with the recent Higgs discovery. Taking the universal soft parameters at the SUSY breaking scale, we obtain the squark mass spectrum at the matching scale where the SM emerges, by using the renormalization group equations (REGs) of the soft masses. On the other hand, the $6 \times 6$ mixing matrix between squarks and quarks is taken to be free at the low energy.

In Section 2, we discuss the squark and gluino mass spectrum and the squark mixing. In Section 3, we present the formulation of the FCNC with $\Delta F=2$ in the $K, B^{0}, B_{s}$ and $D$ meson systems together with nEDM and HgEDM. We present numerical results and discussions in Section 4. Section 5 is devoted to the summary. The relevant formulations are presented in Appendices A-D.

\section{SUSY Spectrum and Squark Mixing}

The low-energy FCNCs depend significantly on the spectrum of the SUSY particles, which depend on the model. As is well known, the lightest Higgs mass can be pushed up to $125 \mathrm{GeV}$ if the squark masses are expected to be $\mathcal{O}(10) \mathrm{TeV}$. Therefore, let us consider the heavy SUSY particle mass spectrum in the framework of the minimal supersymmetric standard model (MSSM), which is consistent with the observed Higgs mass. The discussion of how to obtain the SUSY spectrum has been given in [71,72].

We outline how to obtain the SUSY spectrum in our work. The details are presented in Appendix A. At the SUSY breaking scale $\Lambda$, we write the quadratic terms in the MSSM potential as:

$$
V_{2}=m_{1}^{2}\left|H_{1}\right|^{2}+m_{2}^{2}\left|H_{2}\right|^{2}+m_{3}^{2}\left(H_{1} \cdot H_{2}+\text { h.c. }\right)
$$

Then, the Higgs mass parameter $m^{2}$ is expressed in terms of $m_{1}^{2}, m_{2}^{2}$ and $\tan \beta$ as:

$$
m^{2}=\frac{m_{1}^{2}-m_{2}^{2} \tan ^{2} \beta}{\tan ^{2} \beta-1}
$$

After running down to the $Q_{0}$ scale, in which the SM emerges, by the one-loop SUSY renormalization group equations (RGEs) [73], the scalar potential is the SM one as follows:

$$
V_{S M}=-m^{2}|H|^{2}+\frac{\lambda}{2}|H|^{4}
$$

Here, the Higgs coupling $\lambda$ is given in terms of the SUSY parameters at the leading order as:

$$
\lambda\left(Q_{0}\right)=\frac{1}{4}\left(g^{2}+g^{\prime 2}\right) \cos ^{2} 2 \beta+\frac{3 h_{t}^{2}}{8 \pi^{2}} X_{t}^{2}\left(1-\frac{X_{t}^{2}}{12}\right), \quad X_{t}=\frac{A_{t}\left(Q_{0}\right)-\mu\left(Q_{0}\right) \cot \beta}{Q_{0}}
$$

and $h_{t}$ is the top Yukawa coupling of the SM. The parameters $m_{2}$ and $\lambda$ run with the two-loop SM RGEs with the $\overline{\mathrm{MS}}$ scheme [74-76] down to the electroweak scale $Q_{E W}=m_{H}$ and then give:

$$
m_{H}^{2}=2 m^{2}\left(m_{H}\right)=\lambda\left(m_{H}\right) v^{2}
$$

When $m_{H}=125 \mathrm{GeV}$ is placed, $\lambda\left(Q_{0}\right)$ and $m^{2}\left(Q_{0}\right)$ are obtained. This input constrains the SUSY mass spectrum of the MSSM. In our work, we take the universal soft breaking parameters at the SUSY breaking scale $\Lambda$ as follows:

$$
\begin{aligned}
& m_{\tilde{Q}_{i}}(\Lambda)=m_{\tilde{U}_{i}^{c}}(\Lambda)=m_{\tilde{D}_{i}^{c}}(\Lambda)=m_{\tilde{L}_{i}}(\Lambda)=m_{\tilde{E}_{i}^{c}}(\Lambda)=m_{0}^{2}(i=1,2,3) \\
& M_{1}(\Lambda)=M_{2}(\Lambda)=M_{3}(\Lambda)=m_{1 / 2}, \quad m_{1}^{2}(\Lambda)=m_{2}^{2}(\Lambda)=m_{0}^{2} \\
& A_{U}(\Lambda)=A_{0} y_{U}(\Lambda), \quad A_{D}(\Lambda)=A_{0} y_{D}(\Lambda), \quad A_{E}(\Lambda)=A_{0} y_{E}(\Lambda)
\end{aligned}
$$


By inputting $m_{H}=125 \mathrm{GeV}$ and taking the heavy scalar mass $m_{\mathcal{H}} \simeq Q_{0}$ (see Appendix A), we can obtain the SUSY spectrum for the fixed $Q_{0}$ and $\tan \beta$. The details and numerical results are presented in Appendix A.

Let us consider the squark flavor mixing. As discussed above, there is no flavor mixing at $\Lambda$ in the MSSM. However, in order to consider the non-minimal flavor mixing framework, we allow the off-diagonal components of the squark mass matrices at the $10 \%$ level, which leads to the flavor mixing of order 0.1 . We take these flavor mixing angles as free parameters at low energies. Now, we consider the $6 \times 6$ squark mass matrix $M_{\tilde{q}}$ in the super-CKM basis. In order to move the mass eigenstate basis of squark masses, we should diagonalize the mass matrix by rotation matrix $\Gamma_{G}^{(q)}$ as:

$$
m_{\tilde{q}}^{2}=\Gamma_{G}^{(q)} M_{\tilde{q}}^{2} \Gamma_{G}^{(q) \dagger}
$$

where $\Gamma_{G}^{(q)}$ is the $6 \times 6$ unitary matrix, and we decompose it into the $3 \times 6$ matrices as $\Gamma_{G}^{(q)}=\left(\Gamma_{G L}^{(q)}, \Gamma_{G R}^{(q)}\right)^{T}$ in the following expressions:

$$
\begin{aligned}
\Gamma_{G L}^{(d)} & =\left(\begin{array}{cccccc}
c_{13}^{L} & 0 & s_{13}^{L} e^{-i \phi_{13}^{L}} c_{\theta} & 0 & 0 & -s_{13}^{L} e^{-i \phi_{13}^{L}} s_{\theta} e^{i \phi} \\
-s_{23}^{L} s_{13}^{L} e^{i\left(\phi_{13}^{L}-\phi_{23}^{L}\right)} & c_{23}^{L} & s_{23}^{L} c_{13}^{L} e^{-i \phi_{23}^{L}} c_{\theta} & 0 & 0 & -s_{23}^{L} c_{13}^{L} e^{-i \phi_{23}^{L}} s_{\theta} e^{i \phi} \\
-s_{13}^{L} c_{23}^{L} e^{i \phi_{13}^{L}} & -s_{23}^{L} e^{i \phi_{23}^{L}} & c_{13}^{L} c_{23}^{L} c_{\theta} & 0 & 0 & -c_{13}^{L} c_{23}^{L} s_{\theta} e^{i \phi}
\end{array}\right) \\
\Gamma_{G R}^{(d)} & =\left(\begin{array}{cccccc}
0 & 0 & s_{13}^{R} s_{\theta} e^{-i \phi_{13}^{R} e^{-i \phi}} & c_{13}^{R} & 0 & s_{13}^{R} e^{-i \phi_{13}^{R}} c_{\theta} \\
0 & 0 & s_{23}^{R} c_{13}^{R} s_{\theta} e^{-i \phi_{23}^{R} e^{-i \phi}} & -s_{13}^{R} s_{23}^{R} e^{i\left(\phi_{13}^{R}-\phi_{23}^{R}\right)} & c_{23}^{R} & s_{23}^{R} c_{13}^{R} e^{-i \phi_{23}^{R}} c_{\theta} \\
0 & 0 & c_{13}^{R} c_{23}^{R} s_{\theta} e^{-i \phi} & -s_{13}^{R} c_{23}^{R} e^{i \phi_{13}^{R}} & -s_{23}^{R} e^{i \phi_{23}^{R}} & c_{13}^{R} c_{23}^{R} c_{\theta}
\end{array}\right)
\end{aligned}
$$

where we use abbreviations $c_{i j}^{L, R}=\cos \theta_{i j}^{L, R}, s_{i j}^{L, R}=\sin \theta_{i j}^{L, R}, c_{\theta}=\cos \theta$ and $s_{\theta}=\sin \theta$. Here, $\theta$ is the left-right mixing angle between $\tilde{b}_{L}$ and $\tilde{b}_{R}$, which is discussed in Appendix A. It is remarked that we take $s_{12}^{L, R}=0$ due to the degenerate squark masses of the first and second families, as discussed in Appendix A.

The gluino-squark-quark interaction is given as:

$$
\mathcal{L}_{\text {int }}(\tilde{g} q \tilde{q})=-i \sqrt{2} g_{s} \sum_{\{q\}} \widetilde{q}_{i}^{*}\left(T^{a}\right){\widetilde{G^{a}}}^{a}\left[\left(\Gamma_{G L}^{(q)}\right)_{i j} L+\left(\Gamma_{G R}^{(q)}\right)_{i j} R\right] q_{j}+\text { h.c. }
$$

where $L=\left(1-\gamma_{5}\right) / 2, R=\left(1+\gamma_{5}\right) / 2$ and $\widetilde{G}^{a}$ denotes the gluino field; $q^{i}$ are three left-handed $(i=1,2,3)$ and three right-handed quarks $(i=4,5,6)$. This interaction leads to the gluino-squark-mediated flavor changing processes with $\Delta F=2$ and $\Delta F=1$ through the box and penguin diagrams.

The chargino (neutralino)-squark-quark interaction can be also discussed in a similar way.

\section{FCNC of $\Delta F=2$}

In our previous work [41], we have probed the high-scale SUSY, which is at the $10-50 \mathrm{TeV}$ scale, in the CP violations of $K, B^{0}$ and $B_{s}$ mesons. It is found that $\epsilon_{K}$ is most sensitive to SUSY, even if the SUSY scale is at $50 \mathrm{TeV}$. The SUSY contributions for the time-dependent CP asymmetries of $B^{0}$ and $B_{s}$ with $\Delta B=1$ are suppressed at the SUSY scale of $10 \mathrm{TeV}$. Furthermore, the SUSY contribution 
for the $b \rightarrow s \gamma$ process is also suppressed, since the left-right mixing angle, which induces the chiral enhancement, is very small, as discussed in Appendix A. Therefore, we discuss the neutral meson mixing $P^{0}-\bar{P}^{0}\left(P^{0}=K, B^{0}, B_{s}, D\right)$, which are FCNCs with $\Delta F=2$.

In those FCNCs, the dominant SUSY contribution is given through the gluino-squark interaction. Then, the dispersive part of meson mixing $M_{12}^{P^{0}}\left(P^{0}=K, B^{0}, B_{s}\right)$ is written as:

$$
M_{12}^{P^{0}}=M_{12}^{P^{0}, \mathrm{SM}}+M_{12}^{P^{0}, \mathrm{SUSY}}
$$

where $M_{12}^{q, \text { SUSY }}$ are given by the squark mixing parameters in Equation (8), and its explicit formulation is given in Appendices $\mathrm{B}$ and $\mathrm{C}$.

At first, we discuss the $\Delta B=2$ process, that is the mass differences $\Delta M_{B^{0}}$ and $\Delta M_{B_{s}}$ and the CP-violating phases $\phi_{d}$ and $\phi_{s}$. In general, the contribution of the new physics (NP) to the dispersive part $M_{12}^{q}$ is parameterized as:

$$
M_{12}^{B_{q}}=M_{12}^{q, \mathrm{SM}}+M_{12}^{q, \mathrm{NP}}=M_{12}^{q, \mathrm{SM}}\left(1+h_{q} e^{2 i \sigma_{q}}\right), \quad\left(q=B^{0}, B_{s}\right)
$$

where $M_{12}^{q, \mathrm{NP}}$ are the NP contributions. The generic fits for $B^{0}$ and $B_{s}$ mixing have given the constraints on $\left(h_{q}, \sigma_{q}\right)$ [42], where it is assumed that the NP does not significantly affect the SM tree-level charged-current interaction, that is the absorptive part $\Gamma_{12}^{q}$ is dominated by the decay $b \rightarrow c \bar{c} s$. At present, the NP contributions $h_{q}$ are $10 \%-35 \%$ and $15 \%-25 \%$, depending on $\sigma_{q}$ for $B^{0}$ and $B_{s}$, respectively. Thus, we can expect the sizable NP contribution of $\mathcal{O}(20 \%)$. We will discuss whether the high-scale SUSY can fill in the magnitude of the present NP contribution of $\mathcal{O}(20 \%)$.

Next, we discuss the $\Delta S=2$ process, $\Delta M_{K^{0}}$ and the CP-violating parameter in the $K$ meson, $\epsilon_{K}$. By the similar parametrization in Equation (11), the allowed region of $\left(h_{K}, \sigma_{K}\right)$ has been estimated in [42]. The NP contribution is at least $50 \%$, although there is the strong $\sigma_{K}$ dependence. Therefore, it is important to examine carefully the $\mathrm{CP}$ violating parameter $\epsilon_{K}$, which is given as follows:

$$
\epsilon_{K}=e^{i \phi_{\epsilon}} \sin \phi_{\epsilon}\left(\frac{\operatorname{Im}\left(M_{12}^{K}\right)}{\Delta M_{K}}+\xi\right), \quad \xi=\frac{\operatorname{Im} A_{0}^{K}}{\operatorname{Re} A_{0}^{K}}, \quad \phi_{\epsilon}=\tan ^{-1}\left(\frac{2 \Delta M_{K}}{\Delta \Gamma_{K}}\right)
$$

with $A_{0}^{K}$ being the isospin zero amplitude in $K \rightarrow \pi \pi$ decays. Here, $M_{12}^{K}$ is the dispersive part of the $K^{0}-\bar{K}^{0}$ mixing, and $\Delta M_{K}$ is the mass difference in the neutral $K$ meson. The effects of $\xi \neq 0$ and $\phi_{\epsilon}<\pi / 4$ give a suppression effect in $\epsilon_{K}$, and it is parameterized as $\kappa_{\epsilon}$ and estimated by Buras and Guadagnoli [77] as:

$$
\kappa_{\epsilon}=0.92 \pm 0.02
$$

In the $\mathrm{SM}$, the dispersive part $M_{12}^{K}$ is given as follows,

$$
\begin{aligned}
M_{K}^{12} & =\left\langle K\left|\mathcal{H}_{\Delta F=2}\right| \bar{K}\right\rangle \\
& =-\frac{4}{3}\left(\frac{G_{F}}{4 \pi}\right)^{2} M_{W}^{2} \hat{B}_{K} F_{K}^{2} M_{K}\left(\eta_{c c} \lambda_{c}^{2} E\left(x_{c}\right)+\eta_{t t} \lambda_{t}^{2} E\left(x_{t}\right)+2 \eta_{c t} \lambda_{c} \lambda_{t} E\left(x_{c}, x_{t}\right)\right)
\end{aligned}
$$

where $\lambda_{c}=V_{c s} V_{c d}^{*}, \lambda_{t}=V_{t s} V_{t d}^{*}$. The $E(x)$ 's are the one-loop functions [78], and $\eta_{c c, t t, c t}$ are the QCD corrections [77]. Then, $\left|\epsilon_{K}^{\mathrm{SM}}\right|$ is given in terms of the Wolfenstein parameters $\lambda, \bar{\rho}$ and $\bar{\eta}$ as follows:

$$
\left|\epsilon_{K}^{\mathrm{SM}}\right|=\kappa_{\epsilon} C_{\epsilon} \hat{B}_{K}\left|V_{c b}\right|^{2} \lambda^{2} \bar{\eta}\left(\left|V_{c b}\right|^{2}(1-\bar{\rho}) \eta_{t t} E\left(x_{t}\right)-\eta_{c c} E\left(x_{c}\right)+\eta_{c t} E\left(x_{c}, x_{t}\right)\right)
$$


with

$$
C_{\epsilon}=\frac{G_{F}^{2} F_{K}^{2} m_{K} M_{W}^{2}}{6 \sqrt{2} \pi^{2} \Delta M_{K}}
$$

Note that $\left|\epsilon_{K}^{\mathrm{SM}}\right|$ depends on the non-perturbative parameter $\hat{B}_{K}$ in Equation (15). Recently, the error of this parameter shrank dramatically in the lattice calculations [79]. In our calculation, we use the updated value by the flavor Lattice averaging group [80]:

$$
\hat{B}_{K}=0.766 \pm 0.010
$$

Let us write down $\epsilon_{K}$ as:

$$
\epsilon_{K}=\epsilon_{K}^{\mathrm{SM}}+\epsilon_{K}^{\mathrm{SUSY}}
$$

where $\epsilon_{K}^{\mathrm{SUSY}}$ is induced by the imaginary part of the gluino-squark box diagram, which is presented in Appendices B and C. Since $s_{12}^{L(R)}$ vanishes in our scheme, $\epsilon_{K}^{S U S Y}$ is given in the second order of the squark mixing $s_{13}^{L(R)} \times s_{23}^{L(R)}$.

In addition to the above FCNC processes, the neutron EDM, $d_{n}$, arises through the cEDM of the quarks, $d_{q}^{C}$, due to the gluino-squark mixing [63-68]. By using the QCD sum rules, $d_{n}$ is given as:

$$
d_{n}=\left(0.79 d_{d}-0.20 d_{u}\right)+e\left(0.3 d_{u}^{C}+0.59 d_{d}^{C}\right)
$$

where $d_{q}$ and $d_{q}^{C}$ denote the EDM and cEDM of quarks $d_{q}^{C}$ defined in Appendix D. On the other hand, by using the chiral perturbation theory:

$$
d_{n}=e\left(3.0 d_{u}^{C}+2.5 d_{d}^{C}+0.5 d_{s}^{C}\right)
$$

Therefore, the experimental upper bound [62]:

$$
\left|d_{n}\right|<0.29 \times 10^{-25} \mathrm{ecm}
$$

provides us a strong constraint to the gluino-squark mixing.

The HgEDM can also probe the gluino-squark mixing [70]. The QCD sum rule approach gives [81]:

$$
d_{H g}=e\left(d_{u}^{C}-d_{d}^{C}+0.012 d_{s}^{C}\right) \times 3.2 \times 10^{-2}
$$

and the chiral Lagrangian method gives [82]:

$$
d_{H g}=e\left(d_{u}^{C}-d_{d}^{C}+0.0051 d_{s}^{C}\right) \times 8.7 \times 10^{-3}
$$

The experimental upper bound [69]:

$$
\left|d_{H g}\right|<3.1 \times 10^{-29} \mathrm{ecm}
$$

constrains the gluino-squark mixing.

At the last step, we discuss the charmsector, which is a promising field to probe for the new physics beyond the SM. The $D^{0}-\bar{D}^{0}$ mixing is now well established [83] as follows:

$$
x_{D}=\frac{\Delta M_{D}}{\Gamma_{D}}=(3.6 \pm 1.6) \times 10^{-3}, \quad y_{D}=\frac{\Delta \Gamma_{D}}{2 \Gamma_{D}}=(6.1 \pm 0.7) \times 10^{-3}
$$


where $\Delta M_{D}$ and $\Delta \Gamma_{D}$ are the differences of the masses and the decay widths between the mass eigenstates of the $D$ meson, respectively, and $\Gamma_{D}$ is the averaged decay width of the $D$ meson. Since the SM prediction of $\Delta M_{D}$ at the short distance is much suppressed compared with the experimental value due to the bottom quark loop, the SUSY contribution may be enhanced.

\section{Results and Discussions}

Let us estimate the SUSY contribution of the low-energy FCNC. We calculate the SUSY mass spectrum at $Q_{0}=10,50,100,1000 \mathrm{TeV}$ and interpolate the each mass of the SUSY particle in the region of $Q_{0}=10-1000 \mathrm{TeV}$. This approximation is satisfied within $\mathcal{O}(10 \%)$. Therefore, our numerical results should be taken with the ambiguity of $\mathcal{O}(10 \%)$. The mass spectrum at $Q_{0}=10 \mathrm{TeV}$ is presented in Appendix A. See $[41,60]$ for the mass spectrum at $Q_{0}=50 \mathrm{TeV}$.

Then, we have four mixing angles $\theta_{13}^{L(R)}$ and $\theta_{23}^{L(R)}$, five phase $\phi_{13}^{L(R)}, \phi_{23}^{L(R)}, \phi$. We reduce the number of parameters by taking $\sin \theta_{i j}^{\mathrm{L}}=\sin \theta_{i j}^{\mathrm{R}} \equiv s_{i j}$ for simplicity. In the numerical calculations, we scan the phases of Equation (8) in the region of $0 \sim 2 \pi$ for fixed $s_{i j}$, where the Cabibbo angle 0.22 and the large angle 0.5 are taken as the typical mixing. Other relevant input parameters, such as quark masses $m_{c}$, $m_{b}$, the CKM parameters $V_{u s}, V_{c b}, \bar{\rho}, \bar{\eta}$ and $f_{B}, f_{K}$, etc., have been presented in our previous paper [57], which are referred from the UTfit Collaboration [84] and PDG [62].

\section{1. $B^{0}$ and $B_{s}$ Meson Systems}

At first, we examine the SUSY contribution in the $\Delta B=2$ process. We show the SUSY scale $m_{\tilde{Q}} \equiv Q_{0}$ dependence of the SUSY contributions of $\Delta M_{B^{0}}$ and $\Delta M_{B_{s}}$ in Figure 1a,b, where the experimental central value is shown by the red line. The experimental error bars are the $1 \%$ and $0.1 \%$ levels for $\Delta M_{B^{0}}$ and $\Delta M_{B_{s}}$, respectively. We take $s_{13}=s_{23}=0.22,0.5$. There is no phase dependence in our predictions. It is found that the SUSY contributions in $\Delta M_{B^{0}}$ and $\Delta M_{B_{s}}$ are at most $1.5 \%$ and $0.1 \%$ at $m_{\tilde{Q}}=10 \mathrm{TeV}$, respectively. Namely, the high-scale SUSY cannot explain the NP contributions of $h_{d}=0.1-0.35$ and $h_{s}=0.15-0.25$, which have been discussed in Equation (11). As $m_{\tilde{Q}}$ increases, the SUSY contributions of both $\Delta M_{B^{0}}$ and $\Delta M_{B_{s}}$ decrease approximately with the power of $1 / m_{\tilde{Q}}^{2}$. Thus, there is no hope to observe the SUSY contribution in the $\Delta B=2$ process for the high-scale SUSY. It should be noted that the SM predictions are comparable to these experimental data.

The related phenomena are the CP violations of the non-leptonic decays $B^{0} \rightarrow J / \psi K_{S}$ and $B_{s} \rightarrow$ $J / \psi \phi$. The recent experimental data of these phases are [29,36-38]:

$$
\sin \phi_{d}=0.679 \pm 0.020, \quad \phi_{s}=0.07 \pm 0.09 \pm 0.01
$$

in which the contribution of the gluino-squark-quark interaction may be included. The NP contributions in $\phi_{d}$ and $\phi_{s}$ are expressed in terms of the parameters of Equation (11) as [57]:

$$
\phi_{d}=2 \beta_{d}+\arg \left(1+h_{d} e^{2 i \sigma_{d}}\right), \quad \phi_{s}=-2 \beta_{s}+\arg \left(1+h_{s} e^{2 i \sigma_{s}}\right)
$$

where $\beta_{d}\left(\beta_{s}\right)$ is the one angle of the unitarity triangle giving by the CKM matrix elements of the SM. However, $h_{d}$ and $h_{s}$ in the high-scale SUSY are much suppressed compared with $h_{d}=0.1-0.35$ and 
$h_{s}=0.15-0.25$ of Equation (11), and one cannot find signals of the high-scale SUSY in the CP violating decays $B^{0} \rightarrow J / \psi K_{S}$ and $B_{s} \rightarrow J / \psi \phi$.

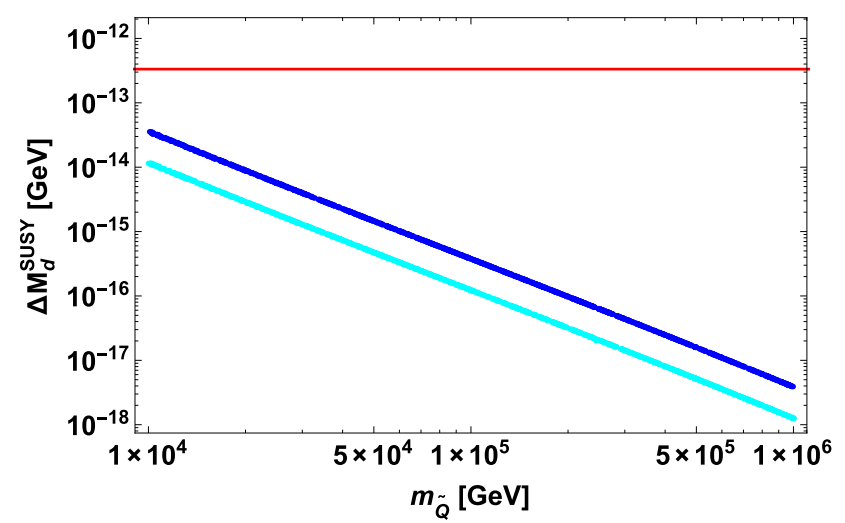

(a)

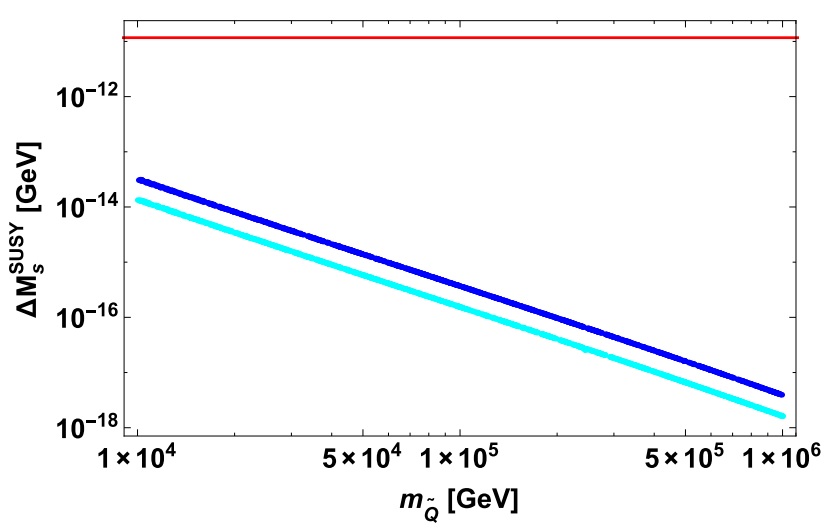

(b)

Figure 1. The SUSY components of (a) $\Delta M_{B^{0}}$ and (b) $\Delta M_{B_{s}}$ versus $m_{\tilde{Q}}$ for $s_{13}=s_{23}=0.22$ (cyan) and 0.5 (blue). The horizontal red line denotes the experimental central value.

\subsection{Neutral K Meson System}

At the second step, we examine the neutral $K$ meson. We show the SUSY contributions of $\Delta M_{K^{0}}$ and $\epsilon_{K}$ versus $m_{\tilde{Q}} \equiv Q_{0}$ in Figure 2a,b, where the experimental central value is shown by the horizontal red line. The experimental error bars are the $0.2 \%$ and $0.5 \%$ levels for $\Delta M_{K^{0}}$ and $\epsilon_{K}$, respectively. Since $\theta_{12}^{L, R}=0$, the SUSY flavor mixing arises from the second order of $s_{13} \times s_{23}$, where $s_{13}=s_{23}=0.22,0.5$ are placed.

It is found in Figure 2a that the SUSY contribution in $\Delta M_{K^{0}}$ can be comparable to the experimental value in the case of $s_{13}=s_{23}=0.5$, whereas it is suppressed in the case of $s_{13}=s_{23}=0.22$ at $m_{\tilde{Q}}=10 \mathrm{TeV}$. Thus, $\Delta M_{K^{0}}$ constrains the squark mixing of $s_{13}$ and $s_{23}$ around $m_{\tilde{Q}}=10 \mathrm{TeV}$. When the SUSY scale increases to more than $20 \mathrm{TeV}$, no SUSY contribution is expected.

On the other hand, $\epsilon_{K}$ is very sensitive to the SUSY contribution up to $100 \mathrm{TeV}$, as seen in Figure $2 \mathrm{~b}$. The plot is scattered due to the random phases of the squark mixing. The experimental data of $\epsilon_{K}$ constrain the squark mixing and phases considerably. Actually, we have already pointed out that the SUSY contribution in $\epsilon_{K}$ could be $40 \%$ and $35 \%$ at $m_{\tilde{Q}}=10,50 \mathrm{TeV}$, respectively [41]. It is found that this sizable SUSY contribution still exist up to $100 \mathrm{TeV}$ in this work.

In the SM, there is only one CP violating phase. Therefore, the observed value of $\phi_{d}$ in Equation (27), should be correlated with $\epsilon_{K}$ in the SM. According to the recent experimental results, it is found that the consistency between the SM prediction and the experimental data of $\sin \phi_{d}$ and $\epsilon_{K}$ is marginal. This fact was pointed out by Buras and Guadagnoli [77] and called the tension between $\epsilon_{K}$ and $\sin \phi_{d}$. Considering the effect of the SUSY contribution $\mathcal{O}(10 \%)$ in $\epsilon_{K}$, this tension can be relaxed even if $m_{\tilde{Q}}=100 \mathrm{TeV}$. The precise determination of the unitarity triangle of $B^{0}$ is required in order to find the SUSY contribution of this level. 
It is noted that the SUSY contribution of both $\Delta M_{K^{0}}$ and $\epsilon_{K}$ also decrease approximately with the power of $1 / m_{\tilde{Q}}^{2}$ as $m_{\tilde{Q}}$ increases up to $1000 \mathrm{TeV}$.

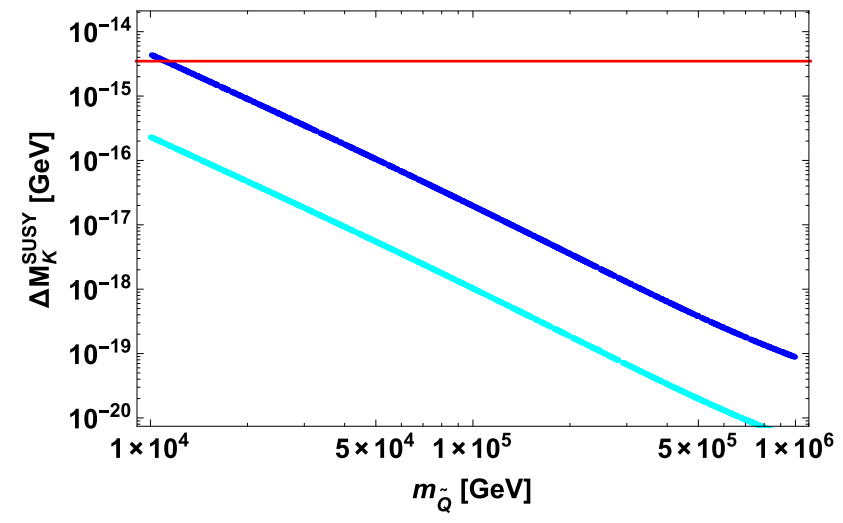

(a)

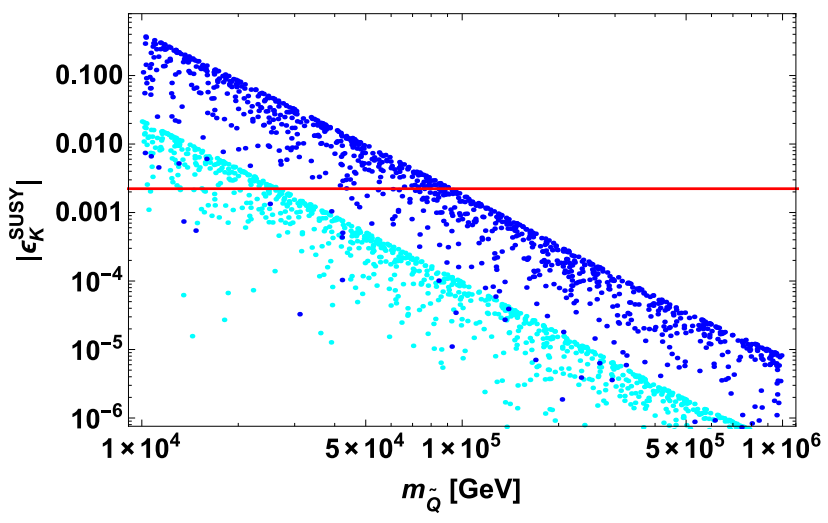

(b)

Figure 2. The SUSY components of (a) $\Delta M_{K^{0}}$ and (b) $\left|\epsilon_{K}\right|$ versus $m_{\tilde{Q}}$ for $s_{13}=s_{23}=0.22$ (cyan) and 0.5 (blue). The horizontal red line denotes the experimental central value.

\subsection{The $n E D M$ and $H g E D M$ with $\epsilon_{K}$}

The nEDM and HgEDM are also sensitive to the SUSY contribution [61,70]. The gluino-squark interaction leads to the cEDM of quarks, which give the nEDM as shown in Equations (19) and (20). We show the predicted nEDM versus $m_{\tilde{Q}}$ for the case of the QCD sum rules of Equation (19) in Figure 3a, where the upper bound of $\left|d_{n}\right|$ is shown by the red line. The plot is scattered due to the random phases of the squark mixing, as well as in the case of $\epsilon_{K}$. We find that the contributions of EDM, $d_{d}$ and $d_{u}$ occupy around $25 \%$ of the neutron EDM. The SUSY contribution is close to the experimental upper bound up to $50 \mathrm{TeV}$. Since the predicted nEDM depends on the phases of the squark mixing matrix significantly, we plot the nEDM versus $\left|\epsilon_{K}^{\mathrm{SUSY}}\right|$ in Figure $3 \mathrm{~b}$. It is found that the predicted nEDM is roughly proportional to $\left|\epsilon_{K}^{\mathrm{SUSY}}\right|$. If the SUSY contribution is the level of $\mathcal{O}(10 \%)$ for $\epsilon_{K}$, the $\mathrm{nEDM}$ is expected to be discovered in the region of $10^{-27}-10^{-26} \mathrm{~cm}$. On the other hand, if the nEDM is not observed above $10^{-28} \mathrm{~cm}$, the SUSY contribution of $\epsilon_{K}$ is below a few \%. Thus, there is the correlation between $d_{n}$ and $\epsilon_{K}^{\mathrm{SUSY}}$.

We also show the predicted HgEDM versus $m_{\tilde{Q}}$ for the case of the QCD sum rules of Equation (22) in Figure $4 a$, where the upper bound of $\left|d_{H g}\right|$ is shown by the red line. The SUSY contribution is close to the experimental upper bound up to $200 \mathrm{TeV}$, which is much higher than the one of the nEDM. In Figure $4 \mathrm{~b}$, we plot the HgEDM versus $\left|\epsilon_{K}^{\mathrm{SUSY}}\right|$. It is found that the experimental upper bound of the HgEDM excludes completely $\left|\epsilon_{K}^{\mathrm{SUSY}}\right|$, which is inconsistent with the experimental data. If the SUSY contribution is the level of $\mathcal{O}(10 \%)$ for $\epsilon_{K}$, the nEDM is expected to be discovered in the region of $10^{-27}-10^{-26} \mathrm{~cm}$. If the HgEDM is not observed above $10^{-29} \mathrm{~cm}$, the SUSY contribution of $\epsilon_{K}$ is below a few \%. Thus, the mercury EDM gives more significant information for the gluino-squark interaction compared with the neutron EDM.

However, these correlations strongly depend on the assumptions of $\theta_{23}^{L}=\theta_{13}^{L}$ and $\theta_{i j}^{L}=\theta_{i j}^{R}$. The deviation from these relations destroys these correlations. For instance, for the case of $\theta_{23}^{L} \gg \theta_{13}^{L}$ with $\theta_{i j}^{L}=\theta_{i j}^{R}, \epsilon_{K}^{\mathrm{SUSY}}$ is much suppressed, whereas the nEDM and HgEDM are still sizable. On the other 
hand, if $\theta_{i j}^{L} \gg \theta_{i j}^{R}$ or $\theta_{i j}^{L} \gg \theta_{i j}^{R}$ is realized, the cEDMs are suppressed, because they require the chirality flipping. In conclusion, careful studies of the mixing angle relations are required to test the correlations between EDMs and $\epsilon_{K}^{\mathrm{SUSY}}$.

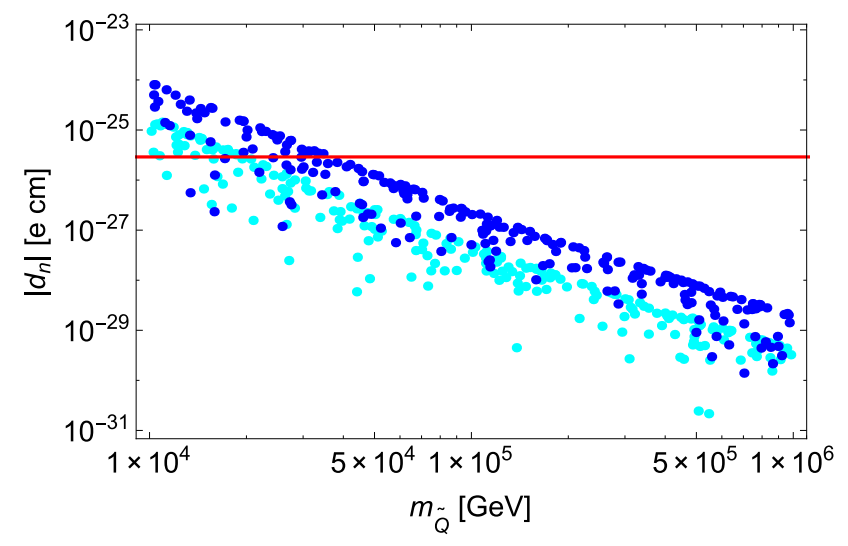

(a)

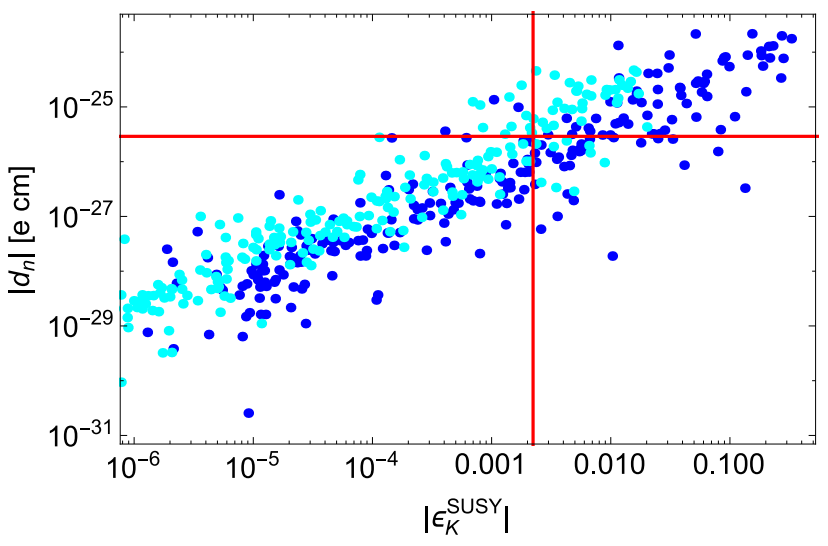

(b)

Figure 3. The the neutron EDM versus (a) $m_{\tilde{Q}}$ and (b) versus $\left|\epsilon_{K}^{\mathrm{SUSY}}\right|$ for $s_{13}=s_{23}=0.22$ (cyan) and 0.5 (blue) for the case of the QCD sum rule. The horizontal red line denotes the experimental upper bound of $\left|d_{n}\right|$, and the vertical one is the experimental central value of $\left|\epsilon_{K}\right|$.

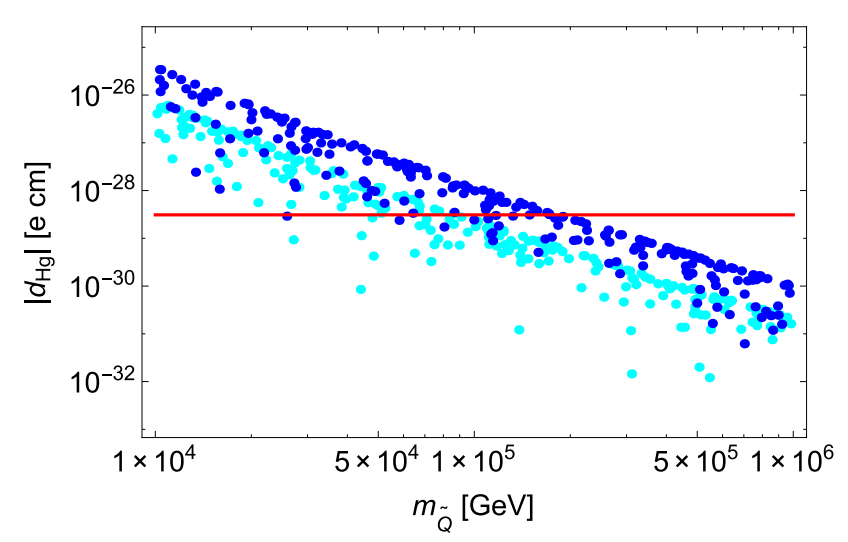

(a)

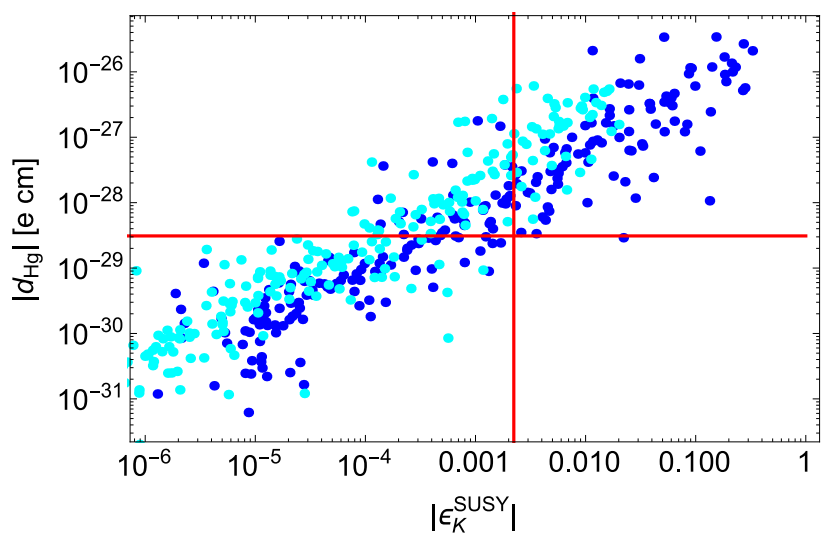

(b)

Figure 4. The the mercury EDM versus (a) $m_{\tilde{Q}}$ and (b) versus $\left|\epsilon_{K}^{\mathrm{SUSY}}\right|$ for $s_{13}=s_{23}=0.22$ (cyan) and 0.5 (blue) for the case of the QCD sum rule. The horizontal red line denotes the experimental upper bound of $\left|d_{H g}\right|$, and the vertical one is the experimental central value of $\left|\epsilon_{K}\right|$.

We should comment on the hadronic model dependence of our numerical result. For both nEDM and $\mathrm{HgEDM}$, we show the numerical result by using the hadronic model of the QCD sum rules in Equations (19) and (22). We have also calculated the EDMs by using the hadronic model of the chiral perturbation theory in Equations (20) and (23). For the neutron EDM, the prediction of the chiral perturbation theory is larger than the one of the QCD sum rule at most of a factor of two. However, for the mercury EDM, the prediction of the QCD sum rule is more than three-times larger compared 
with the one of the chiral perturbation theory. Thus, predicted EDMs have ambiguity with a factor of 2-3 from the hadronic model.

\section{4. $D-\bar{D}$ Mixing}

Since the SM prediction of $\Delta M_{D}$ at the short distance is $\mathcal{O}\left(10^{-18}\right) \mathrm{GeV}$, which is very small compared with the experimental value due to the bottom quark loop, it is important to estimate the SUSY contribution of $\Delta M_{D}$. The mixing angle $\theta_{i j}^{L(R)}$ also appears in the up-type squark mixing matrix, whereas the down-type squark mixing matrix contributes to the $K^{0}, B^{0}$ and $B_{s}$ meson systems induced by the gluino-squark-quark interaction.

We show the SUSY component of $\Delta M_{D}$ and $x_{D}$ versus $m_{\tilde{Q}}$ for $s_{13}=s_{23}=0.22,0.5$ in Figure 5. At the SUSY scale of $10 \mathrm{TeV}$, the SUSY component may be comparable to the observed value. Although the accurate estimate of the long-distance effect is difficult, Cheng and Chiang estimated $x_{D}$ of order $10^{-3}$ from the two body hadronic modes [85]. This obtained value is consistent with the experimental one. Therefore, we should take into account the long-distance effect properly in order to constrain the SUSY contribution from $\Delta M_{D}$.

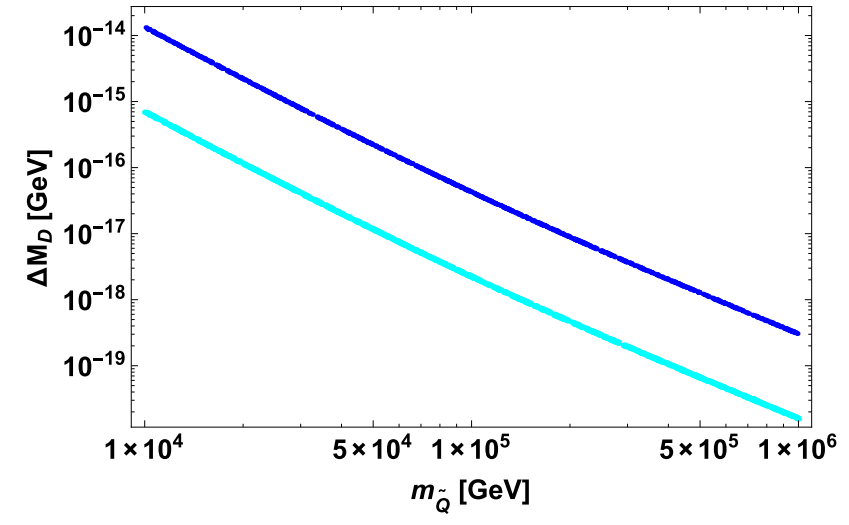

(a)

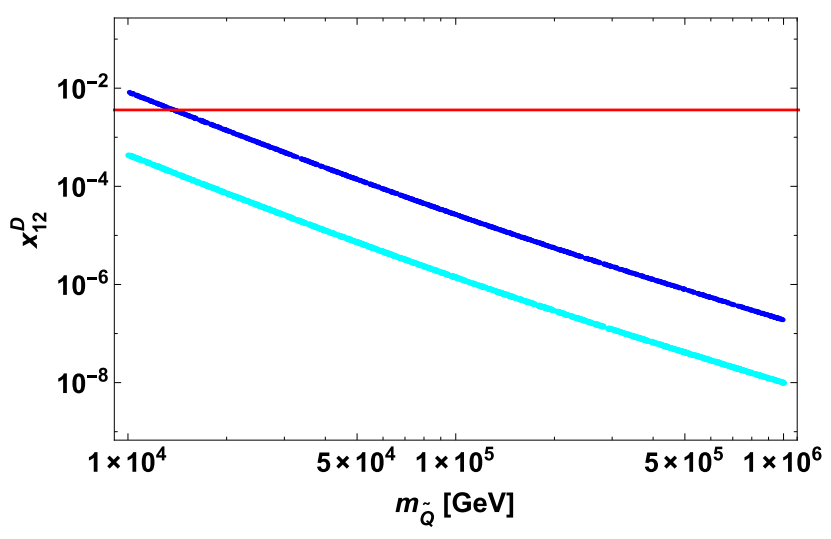

(b)

Figure 5. The SUSY component of (a) $\Delta M_{D}$ and (b) $x_{D}$ versus $m_{\tilde{Q}}$ for $s_{13}=s_{23}=0.22$ (cyan) and 0.5 (blue). The horizontal red line denotes the experimental central value.

Before closing the presentation of the numerical results, we add a comment on the other gaugino contribution. There are additional contributions to the FCNC induced by chargino exchanging diagrams. The chargino contribution to the gluino one is approximately $10 \%$ in the above numerical study of $\Delta F=2$. Thus, the chargino contributions are the sub-leading ones.

\section{Summary}

We discussed the sensitivity of the high-scale SUSY at $10-1000 \mathrm{TeV}$ in the $B^{0}, B_{s}$ and $K^{0}$ meson systems. Furthermore, we have also discussed the sensitivity to the $D-\bar{D}$ mixing, the neutron EDM and the mercury EDM. In order to estimate the contribution of the squark flavor mixing to these FCNC, we calculate the squark mass spectrum, which is consistent with the recent Higgs discovery. 
The SUSY contributions in $\Delta M_{B^{0}}$ and $\Delta M_{B_{s}}$ are at most $1.5 \%$ and $0.1 \%$ at $m_{\tilde{Q}}=10 \mathrm{TeV}$, respectively. As $m_{\tilde{Q}}$ increases, the SUSY contributions of both $\Delta M_{B^{0}}$ and $\Delta M_{B_{s}}$ decrease approximately with the power of $1 / m_{\tilde{Q}}^{2}$. Therefore, the SUSY scale increases to more than $10 \mathrm{TeV}$, and no signal of SUSY is expected. On the other hand, the SUSY contribution in $\Delta M_{K^{0}}$ can be comparable to the experimental value in the case of $s_{13}=s_{23}=0.5$, whereas it is suppressed in the case of $s_{13}=s_{23}=0.22$ at $m_{\tilde{Q}}=10 \mathrm{TeV}$. Furthermore, the SUSY contribution in $\epsilon_{K}$ could be large, around $40 \%$ in the region of the SUSY scale 10-100 TeV. By considering the effect of the SUSY contribution $\mathcal{O}(10 \%)$ in $\epsilon_{K}$, the tension between $\epsilon_{K}$ and $\sin \phi_{d}$ can be relaxed even if the SUSY scale is $100 \mathrm{TeV}$.

The neutron EDM and the mercury EDM are also sensitive to the SUSY contribution induced by the gluino-squark interaction. The $\left|d_{n}\right|$ is expected to be close to the experimental upper bound, even if the SUSY scale is $50 \mathrm{TeV}$. The predicted $\mathrm{nEDM}$ is roughly proportional to $\left|\epsilon_{K}^{\mathrm{SUSY}}\right|$. If the SUSY contribution is the level of $\mathcal{O}(10 \%)$ for $\epsilon_{K}$, the $\left|d_{n}\right|$ is expected to be discovered in the region of $10^{-27}-10^{-26} \mathrm{~cm}$. For the $\left|d_{H g}\right|$, the SUSY contribution is close to the experimental upper bound up to $200 \mathrm{TeV}$, which is much higher than the one of the nEDM. If the HgEDM is not observed above $10^{-29} \mathrm{~cm}$, the SUSY contribution of $\epsilon_{K}$ is below a few \%. Thus, the mercury EDM gives more significant information for the gluino-squark interaction compared with the neutron EDM. It may be important to give a comment that these predictions depend strongly on the assumptions of $\theta_{23}^{L}=\theta_{13}^{L}$ and $\theta_{i j}^{L}=\theta_{i j}^{R}$. The deviation from these relations destroys these correlations. In conclusion, careful studies of the mixing angle relations are required to test the correlations between EDMs and $\epsilon_{K}^{\mathrm{SUSY}}$. The predicted EDMs have also ambiguity with the factor of 2-3 from the hadronic model.

Since the SM prediction of $\Delta M_{D}$ at the short distance is $\mathcal{O}\left(10^{-18}\right) \mathrm{GeV}$, which is very small compared with the experimental value, it is important to estimate the SUSY contribution of $\Delta M_{D}$.

In conclusion, more detailed studies of the $K^{0}$ meson system, the EDMs of the neutron and mercury are required in order to probe the high-scale SUSY at 10-1000 TeV.

\section{Acknowledgments}

This work is supported by JSPS Grant-in-Aid for Scientific Research, 24654062 and 25-5222.

\section{Author Contributions}

Morimitsu Tanimoto and Kei Yamamoto conceived of and discussed ideas of this work, did the calculations and wrote the paper.

\section{Appendix}

\section{A. Running of SUSY Particle Masses}

In the framework of the MSSM, one obtains the SUSY particle spectrum, which is consistent with the observed Higgs mass. The numerical analyses have been given in [71,72]. At the SUSY breaking scale $\Lambda$, the quadratic terms in the MSSM potential are given as:

$$
V_{2}=m_{1}^{2}\left|H_{1}\right|^{2}+m_{2}^{2}\left|H_{2}\right|^{2}+m_{3}^{2}\left(H_{1} \cdot H_{2}+\text { h.c. }\right)
$$


The mass eigenvalues at the $H_{1}$ and $\tilde{H}_{2} \equiv \epsilon H_{2}^{*}$ system are given:

$$
m_{\mp}^{2}=\frac{m_{1}^{2}+m_{2}^{2}}{2} \mp \sqrt{\left(\frac{m_{1}^{2}-m_{2}^{2}}{2}\right)^{2}+m_{3}^{4}}
$$

Suppose that the MSSM matches the SM at the SUSY mass scale $Q_{0} \equiv m_{0}$. Then, the smaller one $m_{-}^{2}$ is identified to be the mass squared of the SM Higgs $H$ with the tachyonic mass. The larger one $m_{+}^{2}$ is the mass squared of the orthogonal combination $\mathcal{H}$, which is decoupled from the SM at $Q_{0}$, that is $m_{\mathcal{H}} \simeq Q_{0}$. Therefore, we have:

$$
m_{-}^{2}=-m^{2}\left(Q_{0}\right), \quad m_{+}^{2}=m_{\mathcal{H}}^{2}\left(Q_{0}\right)=m_{1}^{2}+m_{2}^{2}+m^{2}
$$

with

$$
m_{3}^{4}=\left(m_{1}^{2}+m^{2}\right)\left(m_{2}^{2}+m^{2}\right)
$$

which leads to the mixing angle between $H_{1}$ and $\tilde{H}_{2}, \beta$ as follows:

$$
\tan ^{2} \beta=\frac{m_{1}^{2}+m^{2}}{m_{2}^{2}+m^{2}}, \quad H=\cos \beta H_{1}+\sin \beta \tilde{H}_{2}, \quad \mathcal{H}=-\sin \beta H_{1}+\cos \beta \tilde{H}_{2}
$$

Thus, the Higgs mass parameter $m^{2}$ is expressed in terms of $m_{1}^{2}, m_{2}^{2}$ and $\tan \beta$ :

$$
m^{2}=\frac{m_{1}^{2}-m_{2}^{2} \tan ^{2} \beta}{\tan ^{2} \beta-1}
$$

Below the $Q_{0}$ scale, in which the SM emerges, the scalar potential is the SM one as follows:

$$
V_{S M}=-m^{2}|H|^{2}+\frac{\lambda}{2}|H|^{4}
$$

Here, the Higgs coupling $\lambda$ is given in terms of the SUSY parameters at the leading order as:

$$
\lambda\left(Q_{0}\right)=\frac{1}{4}\left(g^{2}+g^{\prime 2}\right) \cos ^{2} 2 \beta+\frac{3 h_{t}^{2}}{8 \pi^{2}} X_{t}^{2}\left(1-\frac{X_{t}^{2}}{12}\right), \quad X_{t}=\frac{A_{t}\left(Q_{0}\right)-\mu\left(Q_{0}\right) \cot \beta}{Q_{0}}
$$

and $h_{t}$ is the top Yukawa coupling of the SM. The parameters $m_{2}$ and $\lambda$ run with the SM renormalization group equation down to the electroweak scale $Q_{E W}=m_{H}$ and then give:

$$
m_{H}^{2}=2 m^{2}\left(m_{H}\right)=\lambda\left(m_{H}\right) v^{2}
$$

It is easily seen that the VEV of Higgs, $\langle H\rangle$, is $v$, and $\langle\mathcal{H}\rangle=0$, taking account of $\left\langle H_{1}\right\rangle=v \cos \beta$ and $\left\langle H_{2}\right\rangle=v \sin \beta$, where $v=246 \mathrm{GeV}$.

Let us fix $m_{H}=125 \mathrm{GeV}$, which gives $\lambda\left(Q_{0}\right)$ and $m^{2}\left(Q_{0}\right)$. This experimental input constrains the SUSY mass spectrum of the MSSM. We consider the some universal soft breaking parameters at the SUSY breaking scale $\Lambda$ as follows:

$$
\begin{aligned}
& m_{\tilde{Q}_{i}}(\Lambda)=m_{\tilde{U}_{i}^{c}}(\Lambda)=m_{\tilde{D}_{i}^{c}}(\Lambda)=m_{\tilde{L}_{i}}(\Lambda)=m_{\tilde{E}_{i}^{c}}(\Lambda)=m_{0}^{2}(i=1,2,3) \\
& M_{1}(\Lambda)=M_{2}(\Lambda)=M_{3}(\Lambda)=m_{1 / 2} \quad m_{1}^{2}(\Lambda)=m_{2}^{2}(\Lambda)=m_{0}^{2} \\
& A_{U}(\Lambda)=A_{0} y_{U}(\Lambda), \quad A_{D}(\Lambda)=A_{0} y_{D}(\Lambda), \quad A_{E}(\Lambda)=A_{0} y_{E}(\Lambda)
\end{aligned}
$$


Therefore, there is no flavor mixing at $\Lambda$ in the MSSM. However, in order to consider the non-minimal flavor mixing framework, we allow the off-diagonal components of the squark mass matrices at the $10 \%$ level, which leads to the flavor mixing of order 0.1 . We take these flavor mixing angles as free parameters at low energies.

Now, we have the SUSY five parameters, $\Lambda, \tan \beta, m_{0}, m_{1 / 2}, A_{0}$, where $Q_{0}=m_{0}$. In addition to these parameters, we take $\mu=Q_{0}$. Inputting $m_{H}=125 \mathrm{GeV}$ and taking $m_{\mathcal{H}} \simeq Q_{0}$, we can obtain the SUSY spectrum for the fixed $Q_{0}$ and $\tan \beta$.

We present the SUSY mass spectrum at $Q_{0}=10 \mathrm{TeV}$. The input parameter set and the obtained SUSY mass spectra at $Q_{0}$ are summarized in Table A1, where we use $\bar{m}_{t}\left(m_{t}\right)=163.5 \pm 2 \mathrm{GeV}[62,84]$. These parameter sets are easily found from the work in [71].

Table A1. Input parameters at $\Lambda$ and the obtained SUSY spectra at $Q_{0}=10 \mathrm{TeV}$.

\begin{tabular}{cc}
\hline Input at $\boldsymbol{\Lambda}$ and $\boldsymbol{Q}_{\mathbf{0}}$ & Output at $\boldsymbol{Q}_{\mathbf{0}}$ \\
\hline at $\Lambda=10^{17} \mathrm{GeV}$ & $m_{\tilde{g}}=12.8 \mathrm{TeV}, m_{\tilde{W}}=5.2 \mathrm{TeV}, m_{\tilde{B}}=2.9 \mathrm{TeV}$ \\
$m_{0}=10 \mathrm{TeV}$ & $m_{\tilde{b}_{L}}=m_{\tilde{t}_{L}}=12.2 \mathrm{TeV}$ \\
$m_{1 / 2}=6.2 \mathrm{TeV}$ & $m_{\tilde{b}_{R}}=14.1 \mathrm{TeV}, m_{\tilde{t}_{R}}=8.4 \mathrm{TeV}$ \\
$A_{0}=25.803 \mathrm{TeV}$ & $m_{\tilde{s}_{L}, \tilde{d}_{L}}=m_{\tilde{c}_{L}, \tilde{u}_{L}}=15.1 \mathrm{TeV}$ \\
at $Q_{0}=10 \mathrm{TeV}$ & $m_{\tilde{s}_{R}, \tilde{d}_{R}} \simeq m_{\tilde{c}_{R}, \tilde{u}_{R}}=14.6 \mathrm{TeV}, m_{\mathcal{H}}=13.7 \mathrm{TeV}$ \\
$\mu=10 \mathrm{TeV}$ & $m_{\tilde{\tau}_{L}}=m_{{\tilde{\nu_{\tau}}}_{L}}=10.4 \mathrm{TeV}, m_{\tilde{\tau}_{R}}=9.3 \mathrm{TeV}$ \\
$\tan \beta=10$ & $m_{\tilde{\mu}_{L}, \tilde{e}_{L}}=m_{\tilde{\mu}_{L}, \tilde{\nu}_{e}}=10.8 \mathrm{TeV}, m_{\tilde{\mu}_{R}, \tilde{e}_{R}}=10.3 \mathrm{TeV}$ \\
& $X_{t}=-0.22, \quad \lambda_{H}=0.126$ \\
\hline
\end{tabular}

As seen in Table A1, the first and second family squarks are degenerate in their masses; on the other hand, the third ones split due to the large RGE effect. Therefore, the mixing angle between the first and second family squarks vanishes, but the mixing angles between the first-third and the second-third family squarks are produced at the $Q_{0}$ scale. The left-right mixing angle between $\tilde{b}_{L}$ and $\tilde{b}_{R}$ is given as:

$$
\theta \simeq \frac{m_{b}\left(A_{b}\left(Q_{0}\right)-\mu \tan \beta\right)}{m_{\tilde{b}_{L}}^{2}-m_{\tilde{b}_{R}}^{2}}
$$

which is very small, $\mathcal{O}(0.01)$ at $10 \mathrm{TeV}$. The lightest squark is the right-handed stop, and the lightest gaugino is the Bino.

\section{B. Squark Contribution in the $\Delta F=2$ Process}

The $\Delta F=2$ effective Lagrangian from the gluino-sbottom-quark interaction is given as [86]:

$$
\mathcal{L}_{\text {eff }}^{\Delta F=2}=-\frac{1}{2}\left[C_{V L L} O_{V L L}+C_{V R R} O_{V R R}\right]-\frac{1}{2} \sum_{i=1}^{2}\left[C_{S L L}^{(i)} O_{S L L}^{(i)}+C_{S R R}^{(i)} O_{S R R}^{(i)}+C_{S L R}^{(i)} O_{S L R}^{(i)}\right]
$$

where 


$$
\begin{array}{ll}
O_{V L L}=\left(\bar{q}_{a} \gamma^{\mu} L Q^{a}\right)\left(\bar{q}_{b} \gamma^{\mu} L Q^{b}\right), & O_{V R R}=\left(\bar{q}_{a} \gamma^{\mu} R Q^{a}\right)\left(\bar{q}_{b} \gamma^{\mu} R Q^{b}\right) \\
O_{S L L}^{(1)}=\left(\bar{q}_{a} L Q^{a}\right)\left(\bar{q}_{b} L Q^{b}\right), & O_{S L L}^{(2)}=\left(\bar{q}_{a} L Q^{b}\right)\left(\bar{q}_{b} L Q^{a}\right) \\
O_{S R R}^{(1)}=\left(\bar{q}_{a} R Q^{a}\right)\left(\bar{q}_{b} R Q^{b}\right), & O_{S R R}^{(2)}=\left(\bar{q}_{a} R Q^{b}\right)\left(\bar{q}_{b} R Q^{a}\right) \\
O_{S L R}^{(1)}=\left(\bar{q}_{a} L Q^{a}\right)\left(\bar{q}_{b} R Q^{b}\right), & O_{S L R}^{(2)}=\left(\bar{q}_{a} L Q^{b}\right)\left(\bar{q}_{b} R Q^{a}\right)
\end{array}
$$

with $(P, Q, q)=\left(B^{0}, b, d\right),\left(B_{s}, b, s\right),\left(K^{0}, s, d\right)$. The $L, R$ denote $\left(1 \pm \gamma_{5}\right) / 2$, and $a, b$ are color indices. Then, the $P^{0}-\bar{P}^{0}$ mixing, $M_{12}$, is written as:

$$
M_{12}=-\frac{1}{2 m_{P}}\left\langle P^{0}\left|\mathcal{L}_{\text {eff }}^{\Delta F=2}\right| \bar{P}^{0}\right\rangle
$$

The hadronic matrix elements are given in terms of the non-perturbative parameters $B_{i}$ as:

$$
\begin{aligned}
\left\langle P^{0}\left|\mathcal{O}_{V L L}\right| \bar{P}^{0}\right\rangle & =\frac{2}{3} m_{P}^{2} f_{P}^{2} B_{1}, \quad\left\langle P^{0}\left|\mathcal{O}_{V R R}\right| \bar{P}^{0}\right\rangle=\left\langle P^{0}\left|\mathcal{O}_{V L L}\right| \bar{P}^{0}\right\rangle \\
\left\langle P^{0}\left|\mathcal{O}_{S L L}^{(1)}\right| \bar{P}^{0}\right\rangle & =-\frac{5}{12} m_{P}^{2} f_{P}^{2} R_{P} B_{2}, \quad\left\langle P^{0}\left|\mathcal{O}_{S R R}^{(1)}\right| \bar{P}^{0}\right\rangle=\left\langle P^{0}\left|\mathcal{O}_{S L L}^{(1)}\right| \bar{P}^{0}\right\rangle \\
\left\langle P^{0}\left|\mathcal{O}_{S L L}^{(2)}\right| \bar{P}^{0}\right\rangle & =\frac{1}{12} m_{P}^{2} f_{P}^{2} R_{P} B_{3}, \quad\left\langle P^{0}\left|\mathcal{O}_{S R R}^{(2)}\right| \bar{P}^{0}\right\rangle=\left\langle P^{0}\left|\mathcal{O}_{S L L}^{(2)}\right| \bar{P}^{0}\right\rangle \\
\left\langle P^{0}\left|\mathcal{O}_{S L R}^{(1)}\right| \bar{P}^{0}\right\rangle & =\frac{1}{2} m_{P}^{2} f_{P}^{2} R_{P} B_{4}, \quad\left\langle P^{0}\left|\mathcal{O}_{S L R}^{(2)}\right| \bar{P}^{0}\right\rangle=\frac{1}{6} m_{P}^{2} f_{P}^{2} R_{P} B_{5}
\end{aligned}
$$

where

$$
R_{P}=\left(\frac{m_{P}}{m_{Q}+m_{q}}\right)^{2}
$$

The Wilson coefficients for the gluino contribution in Equation (B1) are written as [86]:

$$
\begin{aligned}
& C_{V L L}\left(m_{\tilde{g}}\right)=\frac{\alpha_{s}^{2}}{m_{\tilde{g}}^{2}} \sum_{I, J=1}^{6}\left(\lambda_{G L L}^{(d)}\right)_{I}^{i j}\left(\lambda_{G L L}^{(d)}\right)_{J}^{i j}\left[\frac{11}{18} g_{2[1]}\left(x_{I}^{\tilde{g}}, x_{J}^{\tilde{g}}\right)+\frac{2}{9} g_{1[1]}\left(x_{I}^{\tilde{g}}, x_{J}^{\tilde{g}}\right)\right] \\
& C_{V R R}\left(m_{\tilde{g}}\right)=C_{V L L}\left(m_{\tilde{g}}\right)(L \leftrightarrow R) \\
& C_{S R R}^{(1)}\left(m_{\tilde{g}}\right)=\frac{\alpha_{s}^{2}}{m_{\tilde{g}}^{2}} \sum_{I, J=1}^{6}\left(\lambda_{G L R}^{(d)}\right)_{I}^{i j}\left(\lambda_{G L R}^{(d)}\right)_{J}^{i j} \frac{17}{9} g_{1[1]}\left(x_{I}^{\tilde{g}}, x_{J}^{\tilde{g}}\right) \\
& C_{S L L}^{(1)}\left(m_{\tilde{g}}\right)=C_{S R R}^{(1)}\left(m_{\tilde{g}}\right)(L \leftrightarrow R) \\
& C_{S R R}^{(2)}\left(m_{\tilde{g}}\right)=\frac{\alpha_{s}^{2}}{m_{\tilde{g}}^{2}} \sum_{I, J=1}^{6}\left(\lambda_{G L R}^{(d)}\right)_{I}^{i j}\left(\lambda_{G L R}^{(d)}\right)_{J}^{i j}\left(-\frac{1}{3}\right) g_{1[1]}\left(x_{I}^{\tilde{g}}, x_{J}^{\tilde{g}}\right) \\
& C_{S L L}^{(2)}\left(m_{\tilde{g}}\right)=C_{S R R}^{(2)}\left(m_{\tilde{g}}\right)(L \leftrightarrow R)
\end{aligned}
$$




$$
\begin{aligned}
& C_{S L R}^{(1)}\left(m_{\tilde{g}}\right)=\frac{\alpha_{s}^{2}}{m_{\tilde{g}}^{2}} \sum_{I, J=1}^{6}\left\{\left(\lambda_{G L R}^{(d)}\right)_{I}^{i j}\left(\lambda_{G R L}^{(d)}\right)_{J}^{i j}\left(-\frac{11}{9}\right) g_{2[1]}\left(x_{I}^{\tilde{g}}, x_{J}^{\tilde{g}}\right)\right. \\
&\left.+\left(\lambda_{G L L}^{(d)}\right)_{I}^{i j}\left(\lambda_{G R R}^{(d)}\right)_{J}^{i j}\left[\frac{14}{3} g_{1[1]}\left(x_{I}^{\tilde{g}}, x_{J}^{\tilde{g}}\right)-\frac{2}{3} g_{2[1]}\left(x_{I}^{\tilde{g}}, x_{J}^{\tilde{g}}\right)\right]\right\} \\
& C_{S L R}^{(2)}\left(m_{\tilde{g}}\right)=\frac{\alpha_{s}^{2}}{m_{\tilde{g}}^{2}} \sum_{I, J=1}^{6}\left\{\left(\lambda_{G L R}^{(d)}\right)_{I}^{i j}\left(\lambda_{G R L}^{(d)}\right)_{J}^{i j}\left(-\frac{5}{3}\right) g_{2[1]}\left(x_{I}^{\tilde{g}}, x_{J}^{\tilde{g}}\right)\right. \\
&\left.+\left(\lambda_{G L L}^{(d)}\right)_{I}^{i j}\left(\lambda_{G R R}^{(d)}\right)_{J}^{i j}\left[\frac{2}{9} g_{1[1]}\left(x_{I}^{\tilde{g}}, x_{J}^{\tilde{g}}\right)+\frac{10}{9} g_{2[1]}\left(x_{I}^{\tilde{g}}, x_{J}^{\tilde{g}}\right)\right]\right\}
\end{aligned}
$$

where

$$
\begin{array}{ll}
\left(\lambda_{G L L}^{(d)}\right)_{K}^{i j}=\left(\Gamma_{G L}^{(d) \dagger}\right)_{i}^{K}\left(\Gamma_{G L}^{(d)}\right)_{K}^{j}, & \left(\lambda_{G R R}^{(d)}\right)_{K}^{i j}=\left(\Gamma_{G R}^{(d) \dagger}\right)_{i}^{K}\left(\Gamma_{G R}^{(d)}\right)_{K}^{j} \\
\left(\lambda_{G L R}^{(d)}\right)_{K}^{i j}=\left(\Gamma_{G L}^{(d) \dagger}\right)_{i}^{K}\left(\Gamma_{G R}^{(d)}\right)_{K}^{j}, & \left(\lambda_{G R L}^{(d)}\right)_{K}^{i j}=\left(\Gamma_{G R}^{(d) \dagger}\right)_{i}^{K}\left(\Gamma_{G L}^{(d)}\right)_{K}^{j}
\end{array}
$$

Here, we take $(i, j)=(1,3),(2,3),(1,2)$ which correspond to $B^{0}, B_{s}$ and $K^{0}$ mesons, respectively. The loop functions are given as follows:

- If $x_{I}^{\tilde{g}} \neq x_{J}^{\tilde{g}}\left(x_{I, J}^{\tilde{g}}=m_{\tilde{d}_{I, J}}^{2} / m_{\tilde{g}}^{2}\right)$,

$$
\begin{aligned}
& g_{1[1]}\left(x_{I}^{\tilde{g}}, x_{J}^{\tilde{g}}\right)=\frac{1}{x_{I}^{\tilde{g}}-x_{J}^{\tilde{g}}}\left(\frac{x_{I}^{\tilde{g}} \log x_{I}^{\tilde{g}}}{\left(x_{I}^{\tilde{g}}-1\right)^{2}}-\frac{1}{x_{I}^{\tilde{g}}-1}-\frac{x_{J}^{\tilde{g}} \log x_{J}^{\tilde{g}}}{\left(x_{J}^{\tilde{g}}-1\right)^{2}}+\frac{1}{x_{J}^{\tilde{g}}-1}\right) \\
& g_{2[1]}\left(x_{I}^{\tilde{g}}, x_{J}^{\tilde{g}}\right)=\frac{1}{x_{I}^{\tilde{g}}-x_{J}^{\tilde{g}}}\left(\frac{\left(x_{I}^{\tilde{g}}\right)^{2} \log x_{I}^{\tilde{g}}}{\left(x_{I}^{\tilde{g}}-1\right)^{2}}-\frac{1}{x_{I}^{\tilde{g}}-1}-\frac{\left(x_{J}^{\tilde{g}}\right)^{2} \log x_{J}^{\tilde{g}}}{\left(x_{J}^{\tilde{g}}-1\right)^{2}}+\frac{1}{x_{J}^{\tilde{g}}-1}\right)
\end{aligned}
$$

- If $x_{I}^{\tilde{g}}=x_{J}^{\tilde{g}}$

$$
\begin{aligned}
& g_{1[1]}\left(x_{I}^{\tilde{g}}, x_{I}^{\tilde{g}}\right)=-\frac{\left(x_{I}^{\tilde{g}}+1\right) \log x_{I}^{\tilde{g}}}{\left(x_{I}^{\tilde{g}}-1\right)^{3}}+\frac{2}{\left(x_{I}^{\tilde{g}}-1\right)^{2}} \\
& g_{2[1]}\left(x_{I}^{\tilde{g}}, x_{I}^{\tilde{g}}\right)=-\frac{2 x_{I}^{\tilde{g}} \log x_{I}^{\tilde{g}}}{\left(x_{I}^{\tilde{g}}-1\right)^{3}}+\frac{x_{I}^{\tilde{g}}+1}{\left(x_{I}^{\tilde{g}}-1\right)^{2}}
\end{aligned}
$$

Taking account of the case that the gluino mass is much smaller than the squark mass scale $Q_{0}$, the effective Wilson coefficients are given by using the RGEs for higher-dimensional operators in Equation (B1) at the leading order of QCD as follows:

$$
\begin{aligned}
& C_{V L L}\left(m_{b}(\Lambda=2 \mathrm{GeV})\right)=\eta_{V L L}^{B(K)} C_{V L L}\left(Q_{0}\right) \quad C_{V R R}\left(m_{b}(\Lambda=2 \mathrm{GeV})\right)=\eta_{V R R}^{B(K)} C_{V L L}\left(Q_{0}\right) \\
& \left(\begin{array}{l}
C_{S L L}^{(1)}\left(m_{b}(\Lambda=2 \mathrm{GeV})\right) \\
C_{S L L}^{(2)}\left(m_{b}(\Lambda=2 \mathrm{GeV})\right)
\end{array}\right)=\left(\begin{array}{l}
C_{S L L}^{(1)}\left(Q_{0}\right) \\
C_{S L L}^{(2)}\left(Q_{0}\right)
\end{array}\right) X_{L L}^{-1} \eta_{L L}^{B(K)} X_{L L} \\
& \left(\begin{array}{l}
C_{S R R}^{(1)}\left(m_{b}(\Lambda=2 \mathrm{GeV})\right) \\
C_{S R R}^{(2)}\left(m_{b}(\Lambda=2 \mathrm{GeV})\right)
\end{array}\right)=\left(\begin{array}{l}
C_{S R R}^{(1)}\left(Q_{0}\right) \\
C_{S R R}^{(2)}\left(Q_{0}\right)
\end{array}\right) X_{R R}^{-1} \eta_{R R}^{B(K)} X_{R R} \\
& \left(\begin{array}{l}
C_{S L R}^{(1)}\left(m_{b}(\Lambda=2 \mathrm{GeV})\right) \\
C_{S L R}^{(2)}\left(m_{b}(\Lambda=2 \mathrm{GeV})\right)
\end{array}\right)=\left(\begin{array}{l}
C_{S L R}^{(1)}\left(Q_{0}\right) \\
C_{S L R}^{(2)}\left(Q_{0}\right)
\end{array}\right) X_{L R}^{-1} \eta_{L R}^{B(K)} X_{L R}
\end{aligned}
$$


where

$$
\begin{aligned}
& \eta_{V L L}^{B}=\eta_{V R R}^{B}=\left(\frac{\alpha_{s}\left(Q_{0}\right)}{\alpha_{s}(\tilde{g})}\right)^{\frac{6}{15}}\left(\frac{\alpha_{s}\left(m_{\tilde{g}}\right)}{\alpha_{s}\left(m_{t}\right)}\right)^{\frac{6}{21}}\left(\frac{\alpha_{s}\left(m_{t}\right)}{\alpha_{s}\left(m_{b}\right)}\right)^{\frac{6}{23}} \\
& \eta_{L L}^{B}=\eta_{R R}^{B}=S_{L L}\left(\begin{array}{cc}
\eta_{b \tilde{g}}^{d_{L L}^{1}} & 0 \\
0 & \eta_{b \tilde{g}}^{d_{L L}^{2}}
\end{array}\right) S_{L L}^{-1}, \quad \eta_{L R}^{B}=S_{L R}\left(\begin{array}{cc}
\eta_{b \tilde{g}}^{d_{\tilde{g}}^{1}} & 0 \\
0 & \eta_{b \tilde{g}}^{d_{L R}^{2}}
\end{array}\right) S_{L R}^{-1} \\
& \eta_{b \tilde{g}}=\left(\frac{\alpha_{s}\left(Q_{0}\right)}{\alpha_{s}\left(m_{\tilde{g}}\right)}\right)^{\frac{1}{10}}\left(\frac{\alpha_{s}\left(m_{\tilde{g}}\right)}{\alpha_{s}\left(m_{t}\right)}\right)^{\frac{1}{14}}\left(\frac{\alpha_{s}\left(m_{t}\right)}{\alpha_{s}\left(m_{b}\right)}\right)^{\frac{3}{46}} \\
& \eta_{V L L}^{K}=\eta_{V R R}^{K}=\left(\frac{\alpha_{s}\left(Q_{0}\right)}{\alpha_{s}\left(m_{\tilde{g}}\right)}\right)^{\frac{6}{15}}\left(\frac{\alpha_{s}\left(m_{\tilde{g}}\right)}{\alpha_{s}\left(m_{t}\right)}\right)^{\frac{6}{21}}\left(\frac{\alpha_{s}\left(m_{t}\right)}{\alpha_{s}\left(m_{b}\right)}\right)^{\frac{6}{23}}\left(\frac{\alpha_{s}\left(m_{b}\right)}{\alpha_{s}(\Lambda=2 \mathrm{GeV})}\right)^{\frac{6}{25}} \\
& \eta_{L L}^{K}=\eta_{R R}^{K}=S_{L L}\left(\begin{array}{cc}
\eta_{\Lambda \tilde{g}}^{d_{L}^{1}} & 0 \\
0 & \eta_{\Lambda \tilde{g}}^{d_{L L}^{2}}
\end{array}\right) S_{L L}^{-1}, \quad \eta_{L R}^{K}=S_{L R}\left(\begin{array}{cc}
\eta_{\Lambda \tilde{g}}^{d_{L R}^{1}} & 0 \\
0 & \eta_{\Lambda \tilde{g}}^{d_{L R}^{2}}
\end{array}\right) S_{L R}^{-1} \\
& \eta_{\Lambda \tilde{g}}=\left(\frac{\alpha_{s}\left(Q_{0}\right)}{\alpha_{s}\left(m_{\tilde{g}}\right)}\right)^{\frac{1}{10}}\left(\frac{\alpha_{s}\left(m_{\tilde{g}}\right)}{\alpha_{s}\left(m_{t}\right)}\right)^{\frac{1}{14}}\left(\frac{\alpha_{s}\left(m_{t}\right)}{\alpha_{s}\left(m_{b}\right)}\right)^{\frac{3}{46}}\left(\frac{\alpha_{s}\left(m_{b}\right)}{\alpha_{s}(\Lambda=2 \mathrm{GeV})}\right)^{\frac{3}{50}} \\
& d_{L L}^{1}=\frac{2}{3}(1-\sqrt{241}), \quad d_{L L}^{2}=\frac{2}{3}(1+\sqrt{241}), \quad d_{L R}^{1}=-16, \quad d_{L R}^{2}=2 \\
& S_{L L}=\left(\begin{array}{cc}
\frac{16+\sqrt{241}}{60} & \frac{16-\sqrt{241}}{60} \\
1 & 1
\end{array}\right), \quad S_{L R}=\left(\begin{array}{cc}
-2 & 1 \\
3 & 0
\end{array}\right) \\
& X_{L L}=X_{R R}=\left(\begin{array}{ll}
1 & 0 \\
4 & 8
\end{array}\right), \quad X_{L R}=\left(\begin{array}{cc}
0 & -2 \\
1 & 0
\end{array}\right)
\end{aligned}
$$

For the parameters $B_{i}^{(d)}(i=2-5)$ of $B$ mesons, we use values in [87] as follows:

$$
\begin{array}{ll}
B_{2}^{\left(B_{d}\right)}\left(m_{b}\right)=0.79(2)(4), & B_{3}^{\left(B_{d}\right)}\left(m_{b}\right)=0.92(2)(4) \\
B_{4}^{\left(B_{d}\right)}\left(m_{b}\right)=1.15(3)\left(_{-7}^{+5}\right), & B_{5}^{\left(B_{d}\right)}\left(m_{b}\right)=1.72(4)\left(_{-6}^{+20}\right) \\
B_{2}^{\left(B_{s}\right)}\left(m_{b}\right)=0.80(1)(4), & B_{3}^{\left(B_{s}\right)}\left(m_{b}\right)=0.93(3)(8) \\
B_{4}^{\left(B_{s}\right)}\left(m_{b}\right)=1.16(2)\left(_{-7}^{+5}\right), & B_{5}^{\left(B_{s}\right)}\left(m_{b}\right)=1.75(3)\left(_{-6}^{+21}\right)
\end{array}
$$

On the other hand, we use the most updated values for $\hat{B}_{1}^{(d)}$ and $\hat{B}_{1}^{(s)}$ as [84]:

$$
\hat{B}_{1}^{\left(B_{s}\right)}=1.33 \pm 0.06, \quad \hat{B}_{1}^{\left(B_{s}\right)} / \hat{B}_{1}^{\left(B_{d}\right)}=1.05 \pm 0.07
$$

For the parameters $B_{i}^{K}(i=2-5)$, we use the following values [88],

$$
\begin{aligned}
& B_{2}^{(K)}(2 \mathrm{GeV})=0.66 \pm 0.04, \quad B_{3}^{(K)}(2 \mathrm{GeV})=1.05 \pm 0.12 \\
& B_{4}^{(K)}(2 \mathrm{GeV})=1.03 \pm 0.06, \quad B_{5}^{(K)}(2 \mathrm{GeV})=0.73 \pm 0.10
\end{aligned}
$$

and we take the recent value of Equation (17) for deriving $B_{1}^{(K)}(2 \mathrm{GeV})$.

For the parameters $B_{i}^{D}(i=1-5)$, we use the following values [89,90],

$$
\begin{array}{ll}
B_{1}^{(D)}(3 \mathrm{GeV})=0.75 \pm 0.02, & B_{2}^{(D)}(3 \mathrm{GeV})=0.66 \pm 0.02, \quad B_{3}^{(D)}(3 \mathrm{GeV})=0.96 \pm 0.05, \\
B_{4}^{(D)}(3 \mathrm{GeV})=0.91 \pm 0.04, & B_{5}^{(D)}(3 \mathrm{GeV})=1.10 \pm 0.05 .
\end{array}
$$




\section{The Loop Functions $F_{i}$}

The loop functions $F_{i}\left(x_{\tilde{g}}^{I}\right)$ are given in terms of $x_{\tilde{g}}^{I}=m_{\tilde{g}}^{2} / m_{\tilde{d}_{I}}^{2}(I=3,6)$ as follows:

$$
\begin{aligned}
& F_{1}\left(x_{\tilde{g}}^{I}\right)=\frac{x_{\tilde{g}}^{I} \log x_{\tilde{g}}^{I}}{2\left(x_{\tilde{g}}^{I}-1\right)^{4}}+\frac{\left(x_{\tilde{g}}^{I}\right)^{2}-5 x_{\tilde{g}}^{I}-2}{12\left(x_{\tilde{g}}^{I}-1\right)^{3}}, \quad F_{2}\left(x_{\tilde{g}}^{I}\right)=-\frac{\left(x_{\tilde{g}}^{I}\right)^{2} \log x_{\tilde{g}}^{I}}{2\left(x_{\tilde{g}}^{I}-1\right)^{4}}+\frac{2\left(x_{\tilde{g}}^{I}\right)^{2}+5 x_{\tilde{g}}^{I}-1}{12\left(x_{\tilde{g}}^{I}-1\right)^{3}} \\
& F_{3}\left(x_{\tilde{g}}^{I}\right)=\frac{\log x_{\tilde{g}}^{I}}{\left(x_{\tilde{g}}^{I}-1\right)^{3}}+\frac{x_{\tilde{g}}^{I}-3}{2\left(x_{\tilde{g}}^{I}-1\right)^{2}}, \quad F_{4}\left(x_{\tilde{g}}^{I}\right)=-\frac{x_{\tilde{g}}^{I} \log x_{\tilde{g}}^{I}}{\left(x_{\tilde{g}}^{I}-1\right)^{3}}+\frac{x_{\tilde{g}}^{I}+1}{2\left(x_{\tilde{g}}^{I}-1\right)^{2}}=\frac{1}{2} g_{2[1]}\left(x_{\tilde{g}}^{I}, x_{\tilde{g}}^{I}\right)
\end{aligned}
$$

\section{EDM and Chromo-EDM of Quarks}

We present the EDM of the strange quark from the gluino contribution as the typical example [86]:

$$
d_{s}\left(Q_{0}\right)=-2 \sqrt{4 \pi \alpha\left(m_{\tilde{g}}\right)} \operatorname{Im}\left[A_{s}^{\gamma 22}\left(Q_{0}\right)\right]
$$

where

$$
\begin{aligned}
A_{s}^{\gamma 22}\left(Q_{0}\right)=\frac{Q_{s} \alpha_{s}\left(m_{\tilde{g}}\right)}{4 \pi} & \frac{8}{3} \sum_{I=1}^{6} \frac{1}{2 m_{\tilde{d}_{I}}^{2}}\left\{\left(m_{s}\left(\lambda_{G L L}^{(d)}\right)_{3}^{22}+m_{s}\left(\lambda_{G R R}^{(d)}\right)_{I}^{22}\right)\left(F_{2}\left(x_{\tilde{g}}^{I}\right)\right)\right. \\
& \left.+m_{\tilde{g}}\left(\lambda_{G L R}^{(d)}\right)_{I}^{22}\left(F_{4}\left(x_{\tilde{g}}^{I}\right)\right)\right\}
\end{aligned}
$$

On the other hand, the chromo-EDM (cEDM) of the strange quark from gluino contribution is given as:

$$
d_{s}^{C}\left(Q_{0}\right)=-2 \sqrt{4 \pi \alpha_{s}\left(m_{\tilde{g}}\right)} \operatorname{Im}\left[A_{s}^{g 22}\left(Q_{0}\right)\right]
$$

where

$$
\begin{gathered}
A_{s}^{g 22}\left(Q_{0}\right)=-\frac{\alpha_{s}\left(m_{\tilde{g}}\right)}{4 \pi} \frac{1}{3} \sum_{I=1}^{6} \frac{1}{2 m_{\tilde{d}_{I}}^{2}}\left\{\left(m_{s}\left(\lambda_{G L L}^{(d)}\right)_{I}^{22}+m_{s}\left(\lambda_{G R R}^{(d)}\right)_{I}^{22}\right)\left(9 F_{1}\left(x_{\tilde{g}}^{I}\right)+F_{2}\left(x_{\tilde{g}}^{I}\right)\right)\right. \\
\left.+m_{\tilde{g}}\left(\lambda_{G L R}^{(d)}\right)_{I}^{22}\left(9 F_{3}\left(x_{\tilde{g}}^{I}\right)+F_{4}\left(x_{\tilde{g}}^{I}\right)\right)\right\}
\end{gathered}
$$

Including the RGE effect of QCD [91], the cEDM of the strange quark is given as:

$$
d_{s}^{C}(2 \mathrm{GeV})=d_{s}^{C}\left(Q_{0}\right)\left(\frac{\alpha_{s}\left(Q_{0}\right)}{\alpha_{s}\left(m_{t}\right)}\right)^{\frac{14}{21}}\left(\frac{\alpha_{s}\left(m_{t}\right)}{\alpha_{s}\left(m_{b}\right)}\right)^{\frac{14}{23}}\left(\frac{\alpha_{s}\left(m_{b}\right)}{\alpha_{s}(2 \mathrm{GeV})}\right)^{\frac{14}{25}}
$$

On the other hand, the EDM operator is mixed with the cEDM operator during RGE evolution. Then, one obtains:

$$
\begin{aligned}
& d_{s}(2 \mathrm{GeV})=d_{s}\left(Q_{0}\right)\left(\frac{\alpha_{s}\left(Q_{0}\right.}{\alpha_{s}\left(m_{t}\right)}\right)^{\frac{16}{21}}\left(\frac{\alpha_{s}\left(m_{t}\right)}{\alpha_{s}\left(m_{b}\right)}\right)^{\frac{16}{23}}\left(\frac{\alpha_{s}\left(m_{b}\right)}{\alpha_{s}(2 \mathrm{GeV})}\right)^{\frac{16}{25}}+\frac{8}{g_{s}} d_{s}^{C}\left(Q_{0}\right) \times \\
& {\left[\left(\frac{\alpha_{s}\left(Q_{0}\right.}{\alpha_{s}\left(m_{t}\right)}\right)^{\frac{16}{21}}\left(\frac{\alpha_{s}\left(m_{t}\right)}{\alpha_{s}\left(m_{b}\right)}\right)^{\frac{16}{23}}\left(\frac{\alpha_{s}\left(m_{b}\right)}{\alpha_{s}(2 \mathrm{GeV})}\right)^{\frac{16}{25}}-\left(\frac{\alpha_{s}\left(Q_{0}\right.}{\alpha_{s}\left(m_{t}\right)}\right)^{\frac{14}{21}}\left(\frac{\alpha_{s}\left(m_{t}\right)}{\alpha_{s}\left(m_{b}\right)}\right)^{\frac{14}{23}}\left(\frac{\alpha_{s}\left(m_{b}\right)}{\alpha_{s}(2 \mathrm{GeV})}\right)^{\frac{14}{25}}\right]}
\end{aligned}
$$

The EDMs and cEDMs of the down- and up-quarks induced by the gluino interaction are also given by the similar formulas. 


\section{Conflicts of Interest}

The authors declare no conflict of interest.

\section{References}

1. Aad, G.; Abbott, B.; Abdallah, J.; Abdel Khalek, S.; Abdinov, O.; Aben, R.; Abi, B.; Abolins, M.; AbouZeid, O.S.; Abramowicz, H.; et al. Search for squarks and gluinos with the ATLAS detector in final states with jets and missing transverse momentum using $\sqrt{s}=8 \mathrm{TeV}$ proton-proton collision data. J. High Energy Phys. 2014, 176, 1-52.

2. Chatrchyan, S.; Khachatryan, V.; Sirunyan, A.M.; Tumasyan, A.; Adam, W.; Bergauer, T.; Dragicevic, M.; Ero, J.; Fabjan, C.; Friedl, M.; et al. Search for new physics in the multijet and missing transverse momentum final state in proton-proton collisions at $\sqrt{s}=8 \mathrm{TeV}$. J. High Energy Phys. 2014, 055, doi:10.1007/JHEP06(2014)055.

3. Aad, G.; Abbott, B.; Abdallah, J.; Abdel Khalek, S.; Abdinov, O.; Aben, R.; Abi, B.; Abolins, M.; AbouZeid, O.S.; Abramowicz, H.; et al. Search for top squark pair production in final states with one isolated lepton, jets, and missing transverse momentum in $\sqrt{s}=8 \mathrm{TeV} p p$ collisions with the ATLAS detector. J. High Energy Phys. 2014, 118, doi:10.1140/epjc/s10052-014-3091-0.

4. Aad, G.; Abbott, B.; Abdallah, J.; Abdel Khalek, S.; Abdinov, O.; Aben, R.; Abi, B.; Abolins, M.; AbouZeid, O.S.; Abramowicz, H.; et al. Observation of a new particle in the search for the Standard Model Higgs boson with the ATLAS detector at the LHC. Phys. Lett. B 2012, 716, 1-29.

5. Chatrchyan, S.; Khachatryan, V.; Sirunyan, A.M.; Tumasyan, A.; Adam, W.; Bergauer, T.; Dragicevic, M.; Ero, J.; Fabjan, C.; Friedl, M.; et al. Observation of a new boson at a mass of $125 \mathrm{GeV}$ with the CMS experiment at the LHC. Phys. Lett. B 2012, 716, 30-61.

6. Aad, G.; Abbott, B.; Abdallah, J.; Abdel Khalek, S.; Abdinov, O.; Aben, R.; Abi, B.; Abolins, M.; AbouZeid, O.S.; Abramowicz, H.; et al. Combined Measurement of the Higgs Boson Mass in $p p$ Collisions at $\sqrt{s}=7$ and $8 \mathrm{TeV}$ with the ATLAS and CMS Experiments. 2015, arXiv:1503.07589. arXiv.org e-Print archive. Available online: http://arxiv.org/abs/1503.07589 (accessed on 14 May 2015).

7. Giudice, G.F.; Luty, M.A.; Murayama, H.; Rattazzi, R. Gaugino mass without singlets. J. High Energy Phys. 1998, 027, doi:10.1088/1126-6708/1998/12/027.

8. Wells, J.D. Implications of supersymmetry breaking with a little hierarchy between gauginos and scalars. 2003, arXiv:hep-ph/0306127. arXiv.org e-Print archive. Available online: http://arxiv.org/ abs/hep-ph/0306127 (accessed on 14 May 2015).

9. Arkani-Hamed, N.; Dimopoulos, S. Supersymmetric unification without low energy supersymmetry and signatures for fine-tuning at the LHC. 2004, arXiv:hep-th/0405159. arXiv.org e-Print archive. Available online: http://arxiv.org/abs/hep-th/0405159 (accessed on 14 May 2015).

10. Giudice, G.F.; Romanino, A. Split supersymmetry. Nucl. Phys. B 2004, 699, 65-89.

11. Arkani-Hamed, N.; Dimopoulos, S.; Giudice, G.F.; Romanino, A. Aspects of split supersymmetry. Nucl. Phys. B 2005, 709, 3-46.

12. Wells, J.D. PeV-scale supersymmetry. Phys. Rev. D 2005, 71, doi:10.1103/PhysRevD.71.015013. 
13. Ibe, M.; Moroi, T.; Yanagida, T.T. Possible Signals of Wino LSP at the Large Hadron Collider. Phys. Lett. B 2007, 644, 355-360.

14. Hall, L.J.; Nomura, Y. A Finely-Predicted Higgs Boson Mass from A Finely-Tuned Weak Scale. J. High Energy Phys. 2010, 076, doi:10.1007/JHEP03(2010)076.

15. Hall, L.J.; Nomura, Y. Spread Supersymmetry. J. High Energy Phys. 2012, 082, doi:10.1007/ JHEP01(2012)082.

16. Ibe, M.; Yanagida, T.T. The Lightest Higgs Boson Mass in Pure Gravity Mediation Model. Phys. Lett. B 2012, 374, 374-380.

17. Ibe, M.; Matsumoto, S.; Yanagida, T.T. Pure Gravity Mediation with $m_{3 / 2}=10-100$ TeV. Phys. Rev. D 2012, 85, doi:10.1103/PhysRevD.85.095011.

18. Arvanitaki, A.; Craig, N.; Dimopoulos, S.; Villadoro, G. Mini-Split. J. High Energy Phys. 2013, 126, doi:10.1007/JHEP02(2013)126.

19. Arkani-Hamed, N.; Gupta, A.; Kaplan, D.E.; Weiner, N.; Zorawski, T. Simply Unnatural Supersymmetry. 2012, arXiv:1212.6971. arXiv.org e-Print archive. Available online: http://arxiv.org/abs/1212.6971 (accessed on 14 May 2015).

20. Evans, J.L.; Ibe, M.; Olive, K.A.; Yanagida, T.T. Universality in Pure Gravity Mediation. Eur. Phys. J. C 2013, 73, doi:10.1140/epjc/s10052-013-2468-9.

21. Hisano, J.; Kuwahara, T.; Nagata, N. Grand Unification in High-scale Supersymmetry. Phys. Lett. B 2013, 723, 324-329.

22. Nagata, N.; Otono, H.; Shirai, S. Probing Bino-Gluino Coannihilation at LHC. 2015, arXiv:1504.00504. arXiv.org e-Print archive. Available online: http://arxiv.org/abs/1504.00504 (accessed on 14 May 2015).

23. Altmannshofer, W.; Harnik, R.; Zupan, J. Low Energy Probes of PeV Scale Sfermions. J. High Energy Phys. 2013, 202, doi:10.1007/JHEP11(2013)202.

24. Moroi, T.; Nagai, M. Probing Supersymmetric Model with Heavy Sfermions Using Leptonic Flavor and CP Violations. Phys. Lett. B 2013, 723, 107-112.

25. McKeen, D.; Pospelov, M.; Ritz, A. Electric dipole moment signatures of PeV-scale superpartners. Phys. Rev. D 2013, 87, doi:10.1103/PhysRevD.87.113002.

26. Aaij, R.; Beteta, C.A.; Adeva, B.; Adinolfi, M.; Adrover, C.; Affolder, A.; Ajaltouni, Z.; Albrecht, J.; Alessio, F.; Alexander, M.; et al. Implications of LHCb measurements and future prospects. Eur. Phys. J. C 2013, 73, 1-92.

27. Aaij, R.; Beteta, C.A.; Adeva, B.; Adinolfi, M.; Adrover, C.; Affolder, A.; Ajaltouni, Z.; Albrecht, J.; Alessio, F.; Alexander, M.; et al. First measurement of the CP-violating phase in $B_{s}^{0} \rightarrow \phi \phi$ decays. 2013, arXiv:1303.7125. arXiv.org e-Print archive. Available online: http://arxiv.org/abs/1303.7125 (accessed on 14 May 2015).

28. Aaij, R.; Beteta, C.A.; Adeva, B.; Adinolfi, M.; Adrover, C.; Affolder, A.; Ajaltouni, Z.; Albrecht, J.; Alessio, F.; Alexander, M.; et al. First observation of $C P$ violation in the decays of $B_{s}^{0}$ mesons. 2013, arXiv:1304.6173. arXiv.org e-Print archive. Available online: http://arxiv.org/abs/1304.6173 (accessed on 14 May 2015). 
29. Aaij, R.; Beteta, C.A.; Adeva, B.; Adinolfi, M.; Adrover, C.; Affolder, A.; Ajaltouni, Z.; Albrecht, J.; Alessio, F.; Alexander, M.; et al. Measurement of CP violation and the $B_{s}^{0}$ meson decay width difference with $B_{s}^{0} B_{s}^{0} \rightarrow J / \psi K^{+} K^{-}$and $B_{s}^{0} B_{s}^{0} \rightarrow J / \psi \pi^{+} \pi^{-}$decays. 2013, arXiv:1304.2600. arXiv.org e-Print archive. Available online: http://arxiv.org/abs/1304.2600 (accessed on 14 May 2015).

30. Aaij, R.; Beteta, C.A.; Adeva, B.; Adinolfi, M.; Adrover, C.; Affolder, A.; Ajaltouni, Z.; Albrecht, J.; Alessio, F.; Alexander, M.; et al. Differential branching fraction and angular analysis of the decay $B_{s}^{0} \rightarrow \phi \mu^{+} \mu^{-}$. J. High Energy Phys. 2013, doi:10.1007/JHEP08(2013)131.

31. Aaij, R.; Beteta, C.A.; Adeva, B.; Adinolfi, M.; Adrover, C.; Affolder, A.; Ajaltouni, Z.; Albrecht, J.; Alessio, F.; Alexander, M.; et al. Differential branching fraction and angular analysis of the decay $B^{0} \rightarrow K^{* 0} \mu^{+} \mu^{-}$. 2013, arXiv:1304.6325. arXiv.org e-Print archive. Available online: http://arxiv.org/abs/1304.6325 (accessed on 14 May 2015).

32. Aaij, R.; Beteta, C.A.; Adeva, B.; Adinolfi, M.; Adrover, C.; Affolder, A.; Ajaltouni, Z.; Albrecht, J.; Alessio, F.; Alexander, M.; et al. Precision measurement of the $B_{s}^{0}-\bar{B}_{s}^{0}$ oscillation frequency with the decay $B_{s}^{0} \rightarrow D_{s}^{-} \pi^{+}$. 2013, arXiv:1304.4741. arXiv.org e-Print archive. Available online: http://arxiv.org/abs/1304.4741 (accessed on 14 May 2015).

33. Vesterinen, M. LHCb Semileptonic Asymmetry. 2013, arXiv:1306.0092. arXiv.org e-Print archive. Available online: http://arxiv.org/abs/1306.0092 (accessed on 14 May 2015).

34. Aaij, R.; Beteta, C.A.; Adeva, B.; Adinolfi, M.; Adrover, C.; Affolder, A.; Ajaltouni, Z.; Albrecht, J.; Alessio, F.; Alexander, M.; et al. First evidence for the decay $B^{-} \rightarrow K^{* 0} \mu^{+} \mu^{-}$. 2012, arXiv:1211.2674. arXiv.org e-Print archive. Available online: http://arxiv.org/abs/1211.2674 (accessed on 14 May 2015).

35. Aaij, R.; Beteta, C.A.; Adeva, B.; Adinolfi, M.; Adrover, C.; Affolder, A.; Ajaltouni, Z.; Albrecht, J.; Alessio, F.; Alexander, M.; et al. Measurement of the $C P$ asymmetry in $B^{0} \rightarrow K^{* 0} \mu^{+} \mu^{-}$decays. 2012, arXiv:1210.4492. arXiv.org e-Print archive. Available online: http://arxiv.org/abs/1210.4492 (accessed on 14 May 2015).

36. Amhis, Y.; Banerjee, Sw.; Bernhard, R.; Blyth, S.; Bozek, A.; Bozzi, C.; Carbone, A.; Campos, A.O.; Chistov, R.; Cibinetto, G.; et al. Averages of B-hadron, C-hadron, and tau-lepton properties as of early 2012. 2012, arXiv:1207.1158. arXiv.org e-Print archive. Available online: http://arxiv.org/abs/1207.1158 (accessed on 14 May 2015).

37. Aaij, R.; Beteta, C.A.; Adeva, B.; Adinolfi, M.; Adrover, C.; Affolder, A.; Ajaltouni, Z.; Albrecht, J.; Alessio, F.; Alexander, M.; et al. Measurement of the CP-violating phase $\phi_{s}$ in the decay $B_{s}^{0} \rightarrow J / \psi \phi$. 2011, arXiv:1112.3183. arXiv.org e-Print archive. Available online: http://arxiv.org/abs/1112.3183 (accessed on 14 May 2015).

38. Aaij, R.; Beteta, C.A.; Adeva, B.; Adinolfi, M.; Adrover, C.; Affolder, A.; Ajaltouni, Z.; Albrecht, J.; Alessio, F.; Alexander, M.; et al. Measurement of the CP violating phase $\phi_{s}$ in $\bar{B}_{s}^{0}->J / \psi f_{0}(980)$. 2011, arXiv:1112.3056. arXiv.org e-Print archive. Available online: http://arxiv.org/abs/1112.3056 (accessed on 14 May 2015).

39. Kobayashi, M.; Maskawa, T. CP Violation in the Renormalizable Theory of Weak Interaction. Prog. Theor. Phys. 1973, 49, 652-657. 
40. Gabbiani, F.; Gabrielli, E.; Masiero, A.; Silvestrini, L. A Complete analysis of FCNC and CP constraints in general SUSY extensions of the standard model, Nucl. Phys. B 1996, 477, 321-352.

41. Tanimoto M.; Yamamoto, K. Probing the high scale SUSY in CP violations of $K, B^{0}$ and $B_{s}$ mesons. 2014, arXiv:1404.0520. arXiv.org e-Print archive. Available online: http://arxiv.org/ abs/1404.0520 (accessed on 14 May 2015).

42. Charles, J.; Descotes-Genon, S.; Ligeti, Z.; Monteil, S.; Papucci, M.; Trabelsi, K. Future sensitivity to new physics in $B_{d}, B_{s}$, and $\mathrm{K}$ mixings. Phys. Rev. D 2014, 89, doi:10.1103/PhysRevD.89.033016.

43. King, S.F. Implications of large CP Violation in B mixing for Supersymmetric Standard Models. $J$. High Energy Phys. 2010, 114, doi:10.1007/JHEP09(2010)114.

44. Endo, M.; Shirai, S.; Yanagida, T.T. Split Generation in the SUSY Mass Spectrum and $B_{s}-\bar{B}_{s}$ Mixing. Prog. Theor. Phys. 2011, 125, 921-932.

45. Endo, M.; Yokozaki, N. Large CP Violation in $B_{s}$ Meson Mixing with EDM constraint in Supersymmetry. J. High Energy Phys. 2011, 130, doi:10.1007/JHEP03(2011)130.

46. Kubo, J.; Lenz, A. Large loop effects of extra SUSY Higgs doublets to CP violation in $B^{0}$ mixing. Phys. Rev. D 2010, 82, doi:10.1103/PhysRevD.82.075001.

47. Kaburaki, Y.; Konya, K.; Kubo, J.; Lenz, A. Triangle Relation of Dark Matter, EDM and CP Violation in B0 Mixing in a Supersymmetric Q6 Model. 2010, arXiv:1012.2435. arXiv.org e-Print archive. Available online: http://arxiv.org/abs/1012.2435 (accessed on 14 May 2015).

48. Virto, J. Exact NLO strong interaction corrections to the Delta $F=2$ effective Hamiltonian in the MSSM. 2009, arXiv:0907.5376. arXiv.org e-Print archive. Available online: http://arxiv.org/ abs/0907.5376 (accessed on 14 May 2015).

49. Virto, J. Top mass dependent $O\left(a l p h a_{s}^{3}\right)$ corrections to B-meson mixing in the MSSM. 2011, arXiv:1111.0940. arXiv.org e-Print archive. Available online: http://arxiv.org/abs/1111.0940 (accessed on 14 May 2015).

50. Ko, P.; Park, J.-H. Implications of the measurements of $B_{s}-\bar{B}_{s}$ mixing on SUSY models. 2008, arXiv:0809.0705. arXiv.org e-Print archive. Available online: http://arxiv.org/abs/0809.0705 (accessed on 14 May 2015).

51. Ko, P.; Park, J.-H. Addendum to: Implications of the measurements of $B_{s}-B_{s}$ bar mixing on SUSY models. 2010, arXiv:1006.5821. arXiv.org e-Print archive. Available online: http://arxiv.org/ abs/1006.5821 (accessed on 14 May 2015).

52. Wang, R.M.; Xu, Y.G.; Chang, Q.; Yang, Y.D. Studying of $B_{s}^{0}-\bar{B}_{s}^{0}$ mixing and $B_{s} \rightarrow K^{(*)-} K^{(*)+}$ decays within supersymmetry. Phys. Rev. D 2011, 83, doi:10.1103/PhysRevD.83.095010.

53. Parry, J.K. The Like-sign dimuon charge asymmetry in SUSY models. 2010, arXiv:1006.5331. arXiv.org e-Print archive. Available online: http://arxiv.org/abs/1006.5331 (accessed on 14 May 2015).

54. Hayakawa, A.; Shimizu, Y.; Tanimoto, M.; Yamamoto, K. Squark flavor mixing and CP asymmetry of neutral B mesons at LHCb. Nucl. Phys. B 2012, 710, 446-453.

55. Shimizu, Y.; Tanimoto, M.; Yamamoto, K. Direct CP Violation of $b \rightarrow s \gamma$ and CP Asymmetries of Non-Leptonic B Decays in Squark Flavor Mixing, 2012, arXiv:1205.1705. arXiv.org e-Print archive. Available online: http://arxiv.org/abs/1205.1705 (accessed on 14 May 2015). 
56. Shimizu, Y.; Tanimoto, M.; Yamamoto, K. SUSY contributions to CP violations in $b \rightarrow s$ and $b \rightarrow d$ transitions facing on new data. 2012, arXiv:1212.6486. arXiv.org e-Print archive. Available online: http://arxiv.org/abs/1212.6486 (accessed on 14 May 2015).

57. Shimizu, Y.; Tanimoto, M.; Yamamoto, K. Sensitivity of the squark flavor mixing to the CP violation of $\mathrm{K}, B^{0}$ and $B_{s}$ mesons. 2013, arXiv:1307.0374. arXiv.org e-Print archive. Available online: http://arxiv.org/abs/1307.0374 (accessed on 14 May 2015).

58. Hayakawa, A.; Shimizu, Y.; Tanimoto, M.; Yamamoto, K. Searching for the squark flavor mixing in CP violations of $B_{s}^{-} \rightarrow K^{+} K^{-}$and $K^{0} K^{-0}$ decays. 2013, arXiv:1311.5974. arXiv.org e-Print archive. Available online: http://arxiv.org/abs/1311.5974 (accessed on 14 May 2015).

59. Mescia, F.; Virto, J. Natural SUSY and Kaon Mixing in view of recent results from Lattice QCD. 2012, arXiv:1208.0534. arXiv.org e-Print archive. Available online: http://arxiv.org/abs/1208.0534 (accessed on 14 May 2015).

60. Tanimoto, M.; Yamamoto, K. $K_{L} \rightarrow \pi^{0} \nu \bar{\nu}$ decay correlating with $\epsilon_{K}$ in high-scale SUSY. 2015, arXiv:1503.06270. arXiv.org e-Print archive. Available online: http://arxiv.org/abs/1503.06270 (accessed on 14 May 2015).

61. Fuyuto, K.; Hisano, J.; Nagata, N.; Tsumura, K. QCD Corrections to Quark (Chromo)Electric Dipole Moments in High-scale Supersymmetry. 2013, arXiv:1308.6493. arXiv.org e-Print archive. Available online: http://arxiv.org/abs/1308.6493 (accessed on 14 May 2015).

62. Olive, K.A.; Agashe, K.; Amsler, C.; Antonelli, M.; Arguin, J.-F.; Asner, D.M.; Baer, H.; Band, H.R.; Barnett, R.M.; Basaglia, T.; et al. Review of Particle Physics. Chin. Phys. C 2014, 38, doi:10.1088/1674-1137/38/9/090001.

63. Pospelov, M.; Ritz, A. Neutron EDM from electric and chromoelectric dipole moments of quarks. Phys. Rev. D 2001, 63, doi:10.1103/PhysRevD.63.073015.

64. Hisano, J.; Shimizu, Y. $B \rightarrow \phi K_{s}$ versus electric dipole moment of $\mathrm{Hg}-199$ atom in supersymmetric models with right-handed squark mixing. Phys. Lett. B 2004, 581, 224-230.

65. Hisano, J.; Shimizu, Y. Hadronic electric dipole moments induced by the strangeness and constraints on supersymmetric CP phases. Phys. Rev. D 2004, 70, 093001, doi:10.1103/PhysRevD.70.093001.

66. Hisano, J.; Nagai, M.; Paradisi, P. Flavor effects on the electric dipole moments in supersymmetric theories: A beyond leading order analysis. Phys. Rev. D 2009, 80, 095014, doi:10.1103/PhysRevD.80.095014.

67. Hisano, J.; Lee, J.Y.; Nagata, N.; Shimizu, Y. Reevaluation of Neutron Electric Dipole Moment with QCD Sum Rules. Phys. Rev. D 2012, 85, doi:10.1103/PhysRevD.85.114044.

68. Fuyuto, K.; Hisano, J.; Nagata, N. Neutron Electric Dipole Moment Induced by the Strangeness Revisited. 2012, arXiv:1211.5228. arXiv.org e-Print archive. Available online: http://arxiv.org/abs/1211.5228 (accessed on 14 May 2015).

69. Griffith, W.C.; Swallows, M.D.; Loftus, T.H.; Romalis, M.V.; Heckel, B.R.; Fortson, E.N. Improved Limit on the Permanent Electric Dipole Moment of Hg-199. Phys. Rev. Lett. 2009, 102, 101601, doi:10.1103/PhysRevLett.102.101601. 
70. Chiou, C.C.; Kong, O.C.; Vaidya, R.D. Quark Loop Contributions to Neutron, Deuteron, and Mercury EDMs from Supersymmetry without R parity, 2007, arXiv:0705.3939. arXiv.org e-Print archive. Available online: http://arxiv.org/abs/0705.3939 (accessed on 14 May 2015).

71. Delgado, A.; Garcia, M.; Quiros, M. Electroweak and supersymmetry breaking from the Higgs boson discovery. 2013, arXiv:1312.3235. arXiv.org e-Print archive. Available online: http://arxiv.org/abs/1312.3235 (accessed on 14 May 2015).

72. Giudice, G.F.; Rattazzi, R. Living Dangerously with Low-Energy Supersymmetry. Nucl. Phys. B 2006, 757, 19-46.

73. Martin, S.P. A Supersymmetry primer. 2011, arXiv:9709356. arXiv.org e-Print archive. Available online: http://arxiv.org/pdf/hep-ph/9709356 (accessed on 14 May 2015).

74. Iso, S. What Can We Learn from the $126 \mathrm{GeV}$ Higgs Boson for the Planck Scale Physics? Hierarchy Problem and the Stability of the Vacuum -. 2013, arXiv:1304.0293. arXiv.org e-Print archive. Available online: http://arxiv.org/abs/1304.0293 (accessed on 14 May 2015).

75. Iso, S.; Orikasa, Y. TeV Scale B-L model with a flat Higgs potential at the Planck scale-In view of the hierarchy problem. 2012, arXiv:1210.2848. arXiv.org e-Print archive. Available online: http://arxiv.org/abs/1210.2848 (accessed on 14 May 2015).

76. Bian, L.G. RGE of the Higgs mass in the context of the SM. 2013, arXiv:1303.2402. arXiv.org e-Print archive. Available online: http://arxiv.org/abs/1303.2402 (accessed on 14 May 2015).

77. Buras, A.J.; Guadagnoli, D. Correlations among new CP violating effects in $\Delta F=2$ observables. 2008, arXiv:0805.3887. arXiv.org e-Print archive. Available online: http://arxiv.org/abs/0805.3887 (accessed on 14 May 2015).

78. Inami, T.; Lim, C.S. Effects of Superheavy Quarks and Leptons in Low-Energy Weak Processes $K_{L} \rightarrow \mu \mu^{-}, K^{+} \rightarrow \pi^{+} v v^{-}$and $K^{0} \leftrightarrow K^{-0}$. Prog. Theor. Phys. 1981, 65, 297-314.

79. Bae, T.; Jang, Y.C.; Jeong, H.; Kim, J.; Kim, J.; Kim, K.; Kim, S.; Lee. W.; Leem, J.; Pak, J.; et al. Update on $B_{K}$ and $\varepsilon_{K}$ with staggered quarks. 2013, arXiv:1310.7319. arXiv.org e-Print archive. Available online: http://arxiv.org/abs/1310.7319 (accessed on 14 May 2015).

80. Aoki, S.; Aoki, Y.; Bernard, C.; Blum, T.; Colangelo, G.; Della Morte, M.; Durr, S.; Khadra, A.X.E.; Fukaya, H.; Horsley, R.; et al. Review of lattice results concerning low energy particle physics. 2013, arXiv:1310.8555. arXiv.org e-Print archive. Available online: http://arxiv.org/abs/1310.8555 (accessed on 14 May 2015).

81. Falk, T.; Olive, K.A.; Pospelov, M.; Roiban, R. MSSM predictions for the electric dipole moment of the Hg-199 atom. Nucl. Phys. B 1999, 560, 3-22.

82. Hisano, J.; Kakizaki, M.; Nagai, M.; Shimizu, Y. Hadronic EDMs in SUSY SU(5) GUTs with right-handed neutrinos. Phys. Lett. B 2004, 604, 216-224.

83. Bevan, A.J.; Bona, M.; Ciuchini, M.; Derkach, D.; Franco, E.; Lubicz, V.; Martinelli, G.; Parodi, F.; Pierini, M.; Schiavi, C.; Silvestrini, L.; et al. The UTfit collaboration average of $D$ meson mixing data: Winter 2014. J. High Energy Phys. 2014, 123, doi:10.1007/JHEP03(2014)123.

84. UTfit. Available online: http:/www.utfit.org (accessed on 14 May 2015).

85. Cheng, H.Y.; Chiang, C.W. Long-Distance Contributions to $D^{0}-\bar{D}^{0}$ Mixing Parameters. 2010, arXiv:1005.1106. arXiv.org e-Print archive. Available online: http://arxiv.org/abs/1005.1106 (accessed on 14 May 2015). 
86. Toru Goto. Available online: http://research.kek.jp/people/tgoto/ (accessed on 14 May 2015).

87. Becirevic, D.; Gimenez, V.; Martinelli, G.; Papinutto, M.; Reyes, J. B parameters of the complete set of matrix elements of delta $B=2$ operators from the lattice, J. High Energy Phys. 2002, 025, doi:10.1088/1126-6708/2002/04/025.

88. Allton, C.R.; Conti, L.; Donini, A.; Gimenez, V.; Giusti, L.; Martinelli, G.; Talevi, M.; Vladikas, A. B parameters for Delta $S=2$ supersymmetric operators. Phys. Lett. B 1999, 453, 30-39.

89. Buras, A.J.; Misiak, M.; Urban, J. Two loop QCD anomalous dimensions of flavor changing four quark operators within and beyond the standard model. Nucl. Phys. B 2000, 586, 397-426.

90. Carrasco, N.; Ciuchini, M.; Dimopoulos, P.; Frezzotti, R.; Gimenez, V.; Lubicz, V.; Rossi, G.C.; Sanfilippo, F.; Silvestrini, L.; Simula, S.; et al. $D^{0}-\bar{D}^{0}$ mixing in the standard model and beyond from $N_{f}=2$ twisted mass QCD, Phys. Rev. D 2014, 90, 014502, doi:10.1103/PhysRevD.90.014502.

91. Degrassi, G.; Franco, E.; Marchetti, S.; Silvestrini, L. QCD corrections to the electric dipole moment of the neutron in the MSSM, J. High Energy Phys. 2005, 044, doi:10.1088/1126-6708/ 2005/11/044.

(C) 2015 by the authors; licensee MDPI, Basel, Switzerland. This article is an open access article distributed under the terms and conditions of the Creative Commons Attribution license (http://creativecommons.org/licenses/by/4.0/). 